\title{
EDIpack: A parallel exact diagonalization package for quantum impurity problems
}

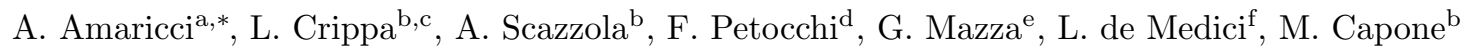 \\ ${ }^{a}$ CNR-IOM, Istituto Officina dei Materiali, Consiglio Nazionale delle Ricerche, Via Bonomea 265, 34136 Trieste, Italy \\ ${ }^{b}$ Scuola Internazionale Superiore di Studi Avanzati (SISSA), Via Bonomea 265, 34136 Trieste, Italy \\ ${ }^{c}$ Institut für Theoretische Physik und Astrophysik and Würzburg-Dresden Cluster of Excellence ct.qmat, Universität \\ Würzburg, 97074 Würzburg, Germany \\ ${ }^{d}$ Department of Physics, University of Fribourg, 1700 Fribourg, Switzerland \\ ${ }^{e}$ Department of Quantum Matter Physics, University of Geneva, Quai Ernest-Ansermet 24, 1211 Geneva, Switzerland \\ ${ }^{f}$ LPEM, ESPCI Paris, PSL Research University, CNRS, Sorbonne Université, 75005 Paris, France
}

\begin{abstract}
We present EDIpack, an exact diagonalization package to solve generic quantum impurity problems. The algorithm includes a generalization of the look-up method introduced in Ref. 1 and enables a massively parallel execution of the matrix-vector linear operations required by Lanczos and Arnoldi algorithms. We show that a suitable Fock basis organization is crucial to optimize the inter-processors communication in a distributed memory setup and to reach sub-linear scaling in sufficiently large systems. We discuss the algorithm in details indicating how to deal with multiple orbitals and electron-phonon coupling. Finally, we outline the download, installation and functioning of the package.
\end{abstract}

Keywords: Exact diagonalization, Quantum Impurity models, Strongly correlated electrons, Dynamical Mean-Field Theory

\section{PROGRAM SUMMARY}

Program Title: EDIpack

CPC Library link to program files: https://doi.org/10.17632/2hxhw9zjg9.1

Code Ocean capsule: https://codeocean.com/capsule/3537659

Licensing provisions: GPLv3

Programming language: Fortran, Python

Classification: $6.5,7.4,20$

Required dependencies: CMake $(>=3.0 .0)$, Scifortran, MPI

Nature of problem: The solution of multi-orbital quantum impurity systems at zero or low temperatures, including the effective description of lattice models of strongly correlated electrons, are difficult to determine.

Solution method: Use a parallel exact diagonalization algorithm to compute the low lying spectrum and evaluate dynamical correlation functions.

\section{Introduction}

The quantum impurity models ${ }^{2}$ play a primary role in the effective description of the local properties of strongly correlated electron systems ${ }^{3}$. Indeed, the reduction of complicated lattice models of correlated electrons to effective impurity systems within dynamical mean-field theory ${ }^{3-6}$ (DMFT) or variational cluster approximations ${ }^{7-10}$ boosted our understanding of correlated materials properties. This scientific advance

\footnotetext{
* Corresponding author.
}

E-mail address: amaricci@sissa.it 
was partially supported by the development of many numerical methods to solve impurity problems within different approximation schemes ${ }^{11,12}$, such as the continuous-time quantum Monte Carlo approaches ${ }^{13-17}$ the numerical renormalization group ${ }^{18-20}$ or the density-matrix renormalization group ${ }^{21-25}$. Among these state-of-the-art methods, the Exact Diagonalization (ED) impurity solver ${ }^{26-31}$ plays a relevant role. The ED method relies on the construction of all or part of the spectrum of a finite quantum system by solving the associated eigenvalue problem, giving access to zero or low temperature properties as well as the exact calculation of one-particle correlation functions on the entire complex plane. This method is not limited to quantum impurity models and the ideas presented in this manuscript can be applied also to a wide range of finite-size quantum systems ${ }^{32,33}$.

In the context of DMFT it has been shown that quantum impurity models with a small Hilbert space already provide accurate results. However, the required size rapidly increases if we consider multi-orbital problems or we include the coupling with phonons. Likewise, larger systems are also required to resolve small energy or temperature scales. The exponential growth of the Hilbert space is the bottleneck of ED calculations. In order to overcome such limitations it is mandatory to take advantage of the blossoming of multi-threaded calculations made available in high-performance computers. In this respect, it is crucial to route the development of ED algorithm for impurity problems in the direction of parallel computing ${ }^{34-36}$. Yet, the parallel formulation of the ED algorithms ${ }^{27,34,36,37}$ can be tremendously more complicated with respect to other methods, e.g. Quantum Monte Carlo, due to the handling of the required inter-processor communications.

In this work we present the implementation of a scalable parallel ED algorithm to solve generic multiorbital quantum impurity problems, including the presence of electron-phonon coupling. The goal of our method is to provide an efficient solver for DMFT calculations with arbitrary electronic band structures at zero or low temperatures, with possible applications to real materials description through $a b$-initio $+\mathrm{DMFT}$ approach $^{38}$. The EDIpack library offers different improvements with respect to the current implementations available in the literature. In order to deal with the multi-orbital structure, we introduce a suitable generalization of the look-up method ${ }^{1,37}$, originally devised for single orbital systems. This generalization takes into account the presence of multiple quantum numbers introducing a great simplification in the construction and organization of the electronic states. In addition, we extend this algorithm to the presence of electron-phonon coupling. In the Fock basis, the Hamiltonian is represented by an exponentially large matrix which makes it impossible to store it, let alone to entirely diagonalize it. The existence of orbital resolved quantum numbers is associated to a product structure of the Hamiltonian matrix, which extends the conventional spin resolution of the hopping terms ${ }^{1,34,36,37}$. The organization of the Hilbert space and the construction of a suitable basis of electronic configurations is a key step in the ED algorithm.

Exploiting the product structure of the Hamiltonian and its sparse nature, i.e. a largely reduced number of finite entries with respect to the total, allows to reduce the memory footprint as well as to employ powerful algorithms designed to access part of the spectrum ${ }^{37,39-42}$. Such methods generally rely on linear operations, like the matrix-vector products, which in turn constitute the largest portion of the computational time. A generic and efficient optimization of these operations is then critical to reduce the computational effort. Here we discuss in details the distributed memory MPI algorithms to perform the required matrix-vector operations. We highlight the bottlenecks created by inter-processor communication congestion in a parallel setup and we show how to unlock a favorable scaling by extending to the multi-orbital and electron-phonon coupled cases the algorithm originally proposed in Ref. 27.

In particular, we show that under very general conditions the scaling of the ED algorithm is (sub)linear in the number of processors, making it possible to solve large systems in a relatively short time and with a moderate distributed memory use. We investigate the effects of multi-orbital interaction on the performances of the parallel algorithms. While nearly optimal scaling can be achieved including only density-density interaction terms ${ }^{43}$, the use of a fully symmetric Kanamori interaction ${ }^{43}$ slightly spoils the scalability of the ED algorithm as a consequence of unavoidable parallel communication blockage. Finally, we highlight that the inclusion of electron-phonon coupling leads to a linear increase of the total execution time, i.e. it reduces by a suitable constant factor the favorable parallel scaling.

The rest of this work is organized as follows: In Sec. 2 we introduce the generic quantum impurity problem and discuss the relevant aspects of the ED method for a generic multi-orbital implementation. In 
Sec. 3 we discuss the parallel algorithms designed to accelerate the execution of the matrix-vector products, at the heart of the ED method. In Sec. 4 we discuss the extension the ED algorithm to the case of electronphonon coupling, including the modifications imposed to the distributed parallelization. Next, in Sec. 5 we discuss in details the scaling properties of this algorithm and their dependence on the number of orbitals and electron-phonon coupling. In Sec. 6 we give an overview of the library structure and discuss the essential aspects of the code implementation. Finally, in Sec. 7 we describe the installation and the use of the library.

\section{Exact Diagonalization}

\subsection{The multi-orbital quantum impurity problem}

We consider a system of $N_{s}$ electronic levels. A portion $N_{\alpha}$ of them, i.e. the impurity levels, interact via a local repulsion, while the remaining $N_{s}-N_{\alpha}$, i.e. the bath levels, are non-interacting. In a typical setup the impurity levels are independently coupled to a set of $N_{b}$ electronic levels, so that the total number is $N_{s}=N_{\alpha}\left(N_{b}+1\right)$. Other choices for the bath topology are possible, which entail a different counting of the total levels. The Hamiltonian of the electronic system we consider has the form:

$$
\begin{aligned}
\hat{H}^{e}= & \hat{H}^{0}+\hat{H}^{i n t} \\
\hat{H}^{0}= & \sum_{\alpha \beta \sigma} H_{\alpha \beta \sigma}^{l o c} d_{\alpha \sigma}^{+} d_{\beta \sigma}+ \\
& \sum_{\nu \alpha \beta \sigma} h_{\alpha \beta \sigma}^{\nu} a_{\nu \alpha \sigma}^{+} a_{\nu \beta \sigma}+\sum_{\nu \alpha \sigma} V_{\alpha \sigma}^{\nu} d_{\alpha \sigma}^{+} a_{\nu \alpha \sigma}+H . c . \\
\hat{H}^{i n t}= & U \sum_{\alpha} n_{\alpha \uparrow} n_{\alpha \downarrow}+U^{\prime} \sum_{\alpha \neq \beta} n_{\alpha \uparrow} n_{\beta \downarrow}+\left(U^{\prime}-J\right) \sum_{\alpha<\beta, \sigma} n_{\alpha \sigma} n_{\beta \sigma}- \\
& J_{X} \sum_{\alpha \neq \beta} d_{\alpha \uparrow}^{+} d_{\alpha \downarrow} d_{\beta \downarrow}^{+} d_{\beta \uparrow}+J_{P} \sum_{\alpha \neq \beta} d_{\alpha \uparrow}^{+} d_{\alpha \downarrow}^{+} d_{\beta \downarrow} d_{\beta \uparrow}
\end{aligned}
$$

where $a_{\alpha \sigma}, d_{\alpha \sigma}\left(a_{\alpha \sigma}^{+}, d_{\alpha \sigma}^{+}\right)$are, respectively, the destruction (creation) operators for the bath and impurity electrons with orbital $\alpha$ and $\operatorname{spin} \sigma, n_{\alpha \sigma}=d_{\alpha \sigma}^{+} d_{\alpha \sigma}$. The term $H_{\alpha \beta \sigma}^{l o c}$ is the non-interacting part of the impurity Hamiltonian, $h_{\alpha \beta \sigma}^{\nu}$ and $V_{\alpha \sigma}^{\nu}$ are, respectively, the local Hamiltonian and the impurity hybridization of the $\nu$-th bath level. Finally, $\hat{H}^{\text {int }}$ is the local multi-orbital interaction ${ }^{43}$. The first three terms represent the density-density part of the interaction, while the remaining two are, respectively, the spin-exchange and pair-hopping terms. $U$ is the local Coulomb interaction strength, $J$ is the Hund's coupling ${ }^{43}$. We introduced independent coupling controlling the spin-exchange and pair-hopping terms, respectively $J_{X}$ and $J_{P}$. The fully symmetric Kanamori interaction is obtained setting $U^{\prime}=U-2 J$ and $J_{X}=J_{P}=J^{43}$.

\subsection{The Fock space and conserved quantum numbers}

A system of $N_{s}$ electrons is associated to a Fock space of the form $\mathcal{F}_{e}=\bigoplus_{n=0}^{N_{s}} S_{-} \mathcal{H}_{e}^{\otimes n}$, where $\mathcal{H}_{e}=$ $\{|0\rangle,|\uparrow\rangle,|\downarrow\rangle,|\uparrow \downarrow\rangle\}$ is the local Hilbert space of the electrons on a single level, $S_{-}$is the anti-symmetrization operator, $\bigoplus$ is the direct sum and $\bigotimes$ the tensor product. The total dimension of the Fock space $\mathcal{F}_{e}$ is $\operatorname{dim}\left(\mathcal{H}_{e}\right)^{N_{s}}=4^{N_{s}}=2^{2 N_{s}}$.

The solution of the eigenvalue problem for $H_{e}$ is simplified by taking into account the existence of conserved quantities. The Hamiltonian (1) conserves the total charge $\hat{N}$ and the spin component $\hat{S}_{z}$, as long as we assume that no terms breaking the particle number or spin conservation are present, e.g. local spin-orbital coupling, in-plane magnetic field, superconducting order, etc. The conservation of $\hat{N}_{\text {and }} \hat{S}_{z}$ can be conveniently re-expressed in terms of conserved number of electrons with spin $\uparrow$ and $\downarrow$, i.e. $\hat{N}_{\uparrow}$, $\hat{N}_{\downarrow}$. Moreover, if the terms $H^{l o c}$ and $h$ are diagonal and we consider only the density-density part of the interaction, i.e. $J_{X}=J_{P}=0$, the number of electrons with spin $\uparrow$ and $\downarrow$ is conserved per orbital, i.e. $\hat{N}_{\uparrow}^{m}$, $\hat{N}_{\downarrow}^{m}$, where $m=1, \ldots, N_{\alpha}$. 
In order to formally unify the treatment of these two cases we introduce some convenient parameters, namely $N_{u d}=1\left(N_{\alpha}\right)$ and $N_{b i t}=N_{s}\left(N_{s} / N_{\alpha}\right)$ for conserved total (orbital resolved) number of electrons with spin $\uparrow$ and $\downarrow$. The first corresponds to the number of conserved operators per spin, the latter indicates the number of electronic levels per spin (spin and orbital). In the following section we will give a more precise meaning to $N_{b i t}$.

In the rest of this work we will indicate the set of conserved quantum numbers $(\mathrm{QN}) \mathrm{s}$ with the tuple $\left[\vec{N}_{\uparrow}, \vec{N}_{\downarrow}\right]$, where $(\sigma=\uparrow, \downarrow)$ :

$$
\vec{N}_{\sigma}= \begin{cases}N_{1 \sigma} \equiv N_{\sigma}, & \text { if } N_{u d}=1 \\ {\left[N_{1 \sigma}, \ldots, N_{N_{\alpha} \sigma}\right],} & \text { if } N_{u d}=N_{\alpha}\end{cases}
$$

Note that for $N_{u d}=1$ we identified the single component $N_{1 \sigma}$ of the vector $\vec{N}_{\sigma}$ with the total number of electrons with spin $\sigma$, i.e. $N_{\sigma}$.

In presence of a set of conserved QNs, the Fock space decomposes into a number of sub-spaces of reduced dimension, each corresponding to a given value of the QNs tuple. We indicate each sub-space with the term sector and the symbol $\mathcal{S}\left[\vec{N}_{\uparrow}, \vec{N}_{\downarrow}\right]$, or just $\mathcal{S}$ where no confusion arises. The dimension of each sector is given by the number of ways we can arrange $N_{\alpha \sigma}$ elements into $N_{b i t}$ positions, i.e.:

$$
\begin{aligned}
\operatorname{dim}\left(\mathcal{S}\left[\vec{N}_{\uparrow}, \vec{N}_{\downarrow}\right]\right) & =\prod_{\alpha=1}^{N_{u d}}\left(\begin{array}{c}
N_{b i t} \\
N_{\alpha \uparrow}
\end{array}\right) \prod_{\alpha=1}^{N_{u d}}\left(\begin{array}{c}
N_{b i t} \\
N_{\alpha \downarrow}
\end{array}\right) \\
& \equiv \prod_{\alpha=1}^{N_{u d}} D_{\alpha \uparrow} \prod_{\alpha=1}^{N_{u d}} D_{\alpha \downarrow} \stackrel{\text { def }}{=} D_{\uparrow} D_{\downarrow} \equiv D_{\mathcal{S}}
\end{aligned}
$$

where we introduced the symbols $D_{\sigma}$ and $D_{\alpha \sigma}$ to indicate, respectively, the dimension of total and orbital spin-subspace associated to the given set of QNs. Note that $D_{\sigma} \equiv D_{1, \sigma}$ for $N_{u d}=1$.

\subsection{The basis states}

A natural representation of the basis states for the Fock space $\mathcal{F}_{e}$ is obtained in the occupation number formalism of second quantization. The Fock basis for a finite system of $N_{s}$ electrons is composed of states of the form $|\vec{n}\rangle=\left|n_{1 \uparrow}, \ldots, n_{N_{s} \uparrow}, n_{1 \downarrow}, \ldots, n_{N_{s} \downarrow}\right\rangle$, where each element $n_{a \sigma}=0,1$ describes the absence or the presence of an electron with spin $\sigma$ at the level $a$. In conjunction with the basis states, we introduce the non-Hermitian, anti-commuting, annihilation and creation operators $c_{a \sigma}$ and $c_{a \sigma}^{+}$, respectively. These operators act on the states $|\vec{n}\rangle$ as:

$$
\begin{aligned}
& c_{a \sigma}|\vec{n}\rangle= \begin{cases}(-1)^{\# a \sigma}\left|\ldots, n_{a \sigma}-1, \ldots\right\rangle & \text { if } n_{a \sigma}=1 \\
0 & \text { otherwise }\end{cases} \\
& c_{a \sigma}^{+}|\vec{n}\rangle= \begin{cases}(-1)^{\# a \sigma}\left|\ldots, n_{a \sigma}+1, \ldots\right\rangle & \text { if } n_{a \sigma}=0 \\
0 & \text { otherwise }\end{cases}
\end{aligned}
$$

with $\#_{a \sigma}=\sum_{b \sigma^{\prime}<a \sigma} n_{b \sigma^{\prime}}$. Thus, each state is represented as a string of zeros and ones, i.e. the binary decomposition of a given integer number $I$. Using the identification $|\vec{n}\rangle=|I\rangle$, with $I=0, \ldots, 2^{2 N_{s}}-1$, each state in the Fock space can be encoded in a computer using a sequence of $2 N_{s}$ bits or, equivalently, an integer number in a fixed representation. The exponentially growing size of the Fock space will eventually make such representation unpractical. A solution is obtained by decomposing each state according to the existing QNs. For a given set $\left[\vec{N}_{\uparrow}, \vec{N}_{\downarrow}\right]$ of QNs we then have:

$$
\begin{aligned}
|\vec{n}\rangle & =\prod_{\alpha=1}^{N_{u d}} \prod_{\sigma=\uparrow \downarrow}\left|n_{1} \ldots n_{N_{b i t}}\right\rangle_{\alpha \sigma} \\
& =\left\{\begin{array}{l}
\left|\vec{n}_{\uparrow}\right\rangle\left|\vec{n}_{\downarrow}\right\rangle, \text { if } N_{u d}=1 \\
\left|\vec{n}_{1 \uparrow}\right\rangle \cdots\left|\vec{n}_{N_{u d} \uparrow}\right\rangle\left|\vec{n}_{1 \downarrow}\right\rangle \cdots\left|\vec{n}_{N_{u d \downarrow} \downarrow}\right\rangle, \text { if } N_{u d}=N_{\alpha}
\end{array}\right.
\end{aligned}
$$


so that, if the total number of electrons with spin $\uparrow$ and $\downarrow$ is conserved, any state is identified by two binary sequences of $N_{b i t}=N_{s}$ bits, one per spin orientation. Alternatively, if the number of electrons with spin $\uparrow$ and $\downarrow$ per orbital is conserved, the states are decomposed into two sets of binary sequences (one set per spin orientation), each sequence being made of $N_{b i t}=N_{s} / N_{\alpha}$ bits. Each binary sequence is associated to a suitable tuple of integer numbers, univocally identifying the Fock state: $I \rightarrow\left[\vec{I}_{\uparrow}, \vec{I}_{\downarrow}\right]$, where $I=0, \ldots, 2^{2 N_{s}}-1$ and $\vec{I}_{\sigma}=\left[I_{1 \sigma}, \ldots, I_{N_{u d} \sigma}\right]$ with $I_{\alpha \sigma}=0, \ldots, 2^{N_{b i t}}-1$. Through such decomposition, each state can be described by the smallest bit set compatible with the conserved QNs. This setup generalizes the method introduced by Lin and Gubernatis in Ref. 1. The relation between the Fock state index $I$ and its tuple decomposition can be easily inverted:

$$
I=I_{1}+\sum_{i=2}^{2 N_{u d}} I_{i} 2^{N_{b i t}(i-1)}
$$

where we rearranged the tuple as $\left[\vec{I}_{\uparrow}, \vec{I}_{\downarrow}\right]=\left[I_{1}, \ldots, I_{2 N_{u d}}\right]$.

Such organization of the Fock states is used to construct a suitable basis for the sectors $\mathcal{S}\left[\vec{N}_{\uparrow}, \vec{N}_{\downarrow}\right]$. To any given Fock state $|\vec{n}\rangle$ and its integer representation $I$, containing the correct bit decomposition dictated by the QNs, it is associated a state $|i\rangle$ and an integer $i=1, \ldots, D_{\mathcal{S}}$ through a suitable map $\overrightarrow{\mathcal{M}}_{\mathcal{S}}$. In particular, each tuple of integers identifying a Fock state belonging to $\mathcal{S}$ is associated to a new tuple specific for each

sector state: $I \in \mathcal{S}=\left[\vec{I}_{\uparrow}, \vec{I}_{\downarrow}\right] \in \mathcal{S} \stackrel{\overrightarrow{\mathcal{M}}_{\mathcal{S}}}{\longrightarrow}\left[\vec{i}_{\uparrow}, \vec{i}_{\downarrow}\right]=i$, where $\vec{i}_{\sigma}=\left[i_{1 \sigma}, \ldots, i_{N_{u d} \sigma}\right]$ and $i_{\alpha \sigma}=1, \ldots, D_{\alpha \sigma}$. The tuple $\left[\vec{i}_{\uparrow}, \vec{i}_{\downarrow}\right]$ univocally identifies a basis state $|i\rangle=\left|\overrightarrow{i_{\uparrow}}, \vec{i}_{\downarrow}\right\rangle$ of the sector $\mathcal{S}$, through a relation similar to (7):

$$
i=i_{1 \uparrow}+\sum_{\sigma} \sum_{l=2}^{N_{u d}}\left(i_{l \sigma}-1\right) \prod_{\alpha=1}^{l-1} D_{\alpha \sigma}
$$

\subsection{The Hamiltonian matrix}

The matrix representing the system Hamiltonian in the Fock space has a block-diagonal structure in presence of a given set of conserved QNs. Each block corresponds to the Hamiltonian of a sector $\mathcal{S}\left[\vec{N}_{\uparrow}, \vec{N}_{\downarrow}\right]$. The analysis of the spectrum, thus, reduces to the recursive analysis of the sector Hamiltonians. In each sector, the Hamiltonian of the system has the following general form:

$$
H_{\mathcal{S}}^{e}=H_{d}+H_{\uparrow} \otimes \rrbracket_{\downarrow}+\rrbracket_{\uparrow} \otimes H_{\downarrow}+H_{n d}
$$

$H_{d}$ is a diagonal term containing the local part of the Hamiltonian, including the density-density terms of the interaction. The $H_{\sigma}$ components describe all the hopping processes of the electrons with spin $\sigma=\uparrow, \downarrow$. Finally the term $H_{n d}$ contains all the remaining non-diagonal elements which do not fit in the previous two components, e.g. the spin-exchange and pair-hopping interaction terms. If the QNs are conserved per orbital, i.e. if $H_{n d} \equiv 0$ and no inter-orbital local hopping terms are present, each $H_{\sigma}$ further splits into a sum of smaller terms:

$$
H_{\sigma}=\sum_{\alpha=1}^{N_{\alpha}} \rrbracket_{1 \sigma} \otimes \cdots \otimes H_{\alpha \sigma} \otimes \cdots \otimes \rrbracket_{N_{\alpha} \sigma}
$$

Each term of the Hamiltonian matrix is constructed independently iterating over one or more components of the sector basis states. For example, the construction of the matrices $H_{\alpha \sigma}$ only involve iterations over the components $\left|i_{\alpha \sigma}\right\rangle$ of the sector basis. In general we have:

$$
\begin{aligned}
\left\langle\vec{i}_{\uparrow} \vec{i}_{\downarrow}\left|H_{\mathcal{S}}^{e}\right| \vec{j}_{\uparrow} \vec{j}_{\downarrow}\right\rangle= & \left\langle\vec{i}_{\uparrow} \vec{i}_{\downarrow}\left|H_{d}+H_{\uparrow} \otimes \square_{\downarrow}+\square_{\uparrow} \otimes H_{\downarrow}+H_{n d}\right| \vec{j}_{\uparrow} \vec{j}_{\downarrow}\right\rangle \\
= & \left\langle\vec{i}_{\uparrow} \vec{i}_{\downarrow}\left|H_{d}\right| \vec{i}_{\uparrow} \vec{i}_{\downarrow}\right\rangle+ \\
& \left\langle\vec{i}_{\uparrow}\left|H_{\uparrow}\right| \vec{j}_{\uparrow}\right\rangle \delta_{\vec{i}_{\downarrow} \vec{j}_{\downarrow}}+\left\langle\vec{i}_{\downarrow}\left|H_{\downarrow}\right| \vec{j}_{\downarrow}\right\rangle \delta_{\vec{i}_{\uparrow} \vec{j}_{\uparrow}}+ \\
& \left\langle\vec{i}_{\uparrow} \vec{i}_{\downarrow}\left|H_{n d}\right| \vec{j}_{\uparrow} \vec{j}_{\downarrow}\right\rangle
\end{aligned}
$$


For very large systems, storing the Hamiltonian matrix in the memory can be highly inefficient. In such cases, Krylov or Lanczos methods ${ }^{1,39,44,45}$ can be implemented using a storage-free algorithm, performing the necessary linear operations on-the-fly. This solution has generally a negative impact on the execution time, however this can be well compensated by scaling in a massively parallel framework.

\section{Matrix-vector product: parallel algorithms}

The bottleneck of any Lanczos based diagonalization is the execution of matrix-vector product (MVP) operations, which often takes up to $90 \%$ of the execution time. For any given sector $\mathcal{S}$ the MVP of the Hamiltonian with an arbitrary vector is $|w\rangle=H_{\mathcal{S}}^{e}|v\rangle$ or, projecting onto the sector basis, $w_{i}=\sum_{j}\left[H_{\mathcal{S}}^{e}\right]_{i j} v_{j}$. Distributing the burden of the MVP across multiple processors can dramatically improve ED calculations and unlock access to larger systems. Yet, the MVP heavily relies on communication among different processors, making the design of efficient parallel algorithms a non-trivial task. In the following, we discuss algorithms based on the distributed memory Message Passing Interface (MPI) framework.

\subsection{The MPI_AllGatherV algorithm}

A simple and generic parallel algorithm is constructed as follows. Let us consider the expansion of the vector $|v\rangle$ onto the sector basis $|v\rangle=\sum_{i} v_{i}|i\rangle$. The vector elements $v_{i}$ are distributed across the processes in data chunks of $\mathrm{Q}=D_{\mathcal{S}} / \mathrm{p}+\mathrm{R}$ length, with $\mathrm{R}=\bmod \left(D_{\mathcal{S}}, \mathrm{p}\right)$ for the first $\mathrm{CPU}$ and $\mathrm{R}=0$ otherwise. In order to distribute the MVP operation among the processes, we assign a share of $\mathrm{Q}$ rows of the matrix $H_{\mathcal{S}}^{e}$ to each process, consistently with the splitting of the vector $|v\rangle$. In doing that, it is important to distinguish between local and non-local elements of the vector, i.e. the share of the vector which resides in the memory of a given process and the remaining parts which live on the memory of the other processes.

As such, the result of the MVP consists of two contributions. The first comes from the product of the $\mathrm{Q} \times \mathbf{Q}$ diagonal blocks of $H_{\mathcal{S}}^{e}$ and the local parts of the vector $|v\rangle$. This term of the product is executed locally on each process, i.e. without any inter-process communication. The second contribution comes from the multiplication of the remaining elements of the matrix share and the vector. Due to its non-local nature, this term of the MVP requires to reconstruct the distributed vector $|v\rangle$. This task is achieved through a call to the MPI function MPI_AllGatherV, which executes a communication of the vector shares from each process to all the other processes.

Although conceptually simple, the scaling analysis of this algorithm reveals a quick saturation already for a number $\mathrm{p}$ of processors of the order of ten. Indeed, the gain deriving from the decreased size $\mathrm{Q}$ of the vector share $(\simeq 1 / \mathrm{p})$ is rapidly balanced and overcome by the inter-processors communication in MPI_AllGatherV, which amount to a massive data transfer among processors. Such communication congestion ultimately prevents to achieve a good parallel scaling.

\subsection{The MPI_AllToAllV algorithm}

An improved, yet less generic, parallel algorithm can be devised exploiting the basis states decomposition introduced above ${ }^{27}$. In the sector basis $\left|\vec{i}_{\uparrow} \vec{i}_{\downarrow}\right\rangle$ a generic vector $|v\rangle$ can be decomposed as:

$$
|v\rangle=\sum_{i} v_{i}|i\rangle=\sum_{\vec{i}_{\uparrow} \vec{i}_{\downarrow}} v_{\vec{i}_{\uparrow}, \vec{i}_{\downarrow}}\left|\vec{i}_{\uparrow} \vec{i}_{\downarrow}\right\rangle=\sum_{i_{\uparrow}=1}^{D_{\uparrow}} \sum_{i_{\downarrow}=1}^{D_{\downarrow}} v_{i_{\uparrow}, i_{\downarrow}}\left|i_{\uparrow}\right\rangle\left|i_{\downarrow}\right\rangle
$$

where in the last equality we used the relation (8) to rearrange the tuples of indices $\vec{i}_{\uparrow}, \vec{i}_{\downarrow}$ and the states $\left|\vec{i}_{\uparrow} \vec{i}_{\downarrow}\right\rangle$ in, respectively, a pair of scalar indices $i_{\uparrow}, i_{\downarrow}$ and states of the form $\left|i_{\uparrow}\right\rangle\left|i_{\downarrow}\right\rangle$. The sector basis states are ordered so that the spin- $\uparrow$ index runs faster than the spin- $\downarrow$ one. Thus, any vector $|v\rangle$ can be represented as 

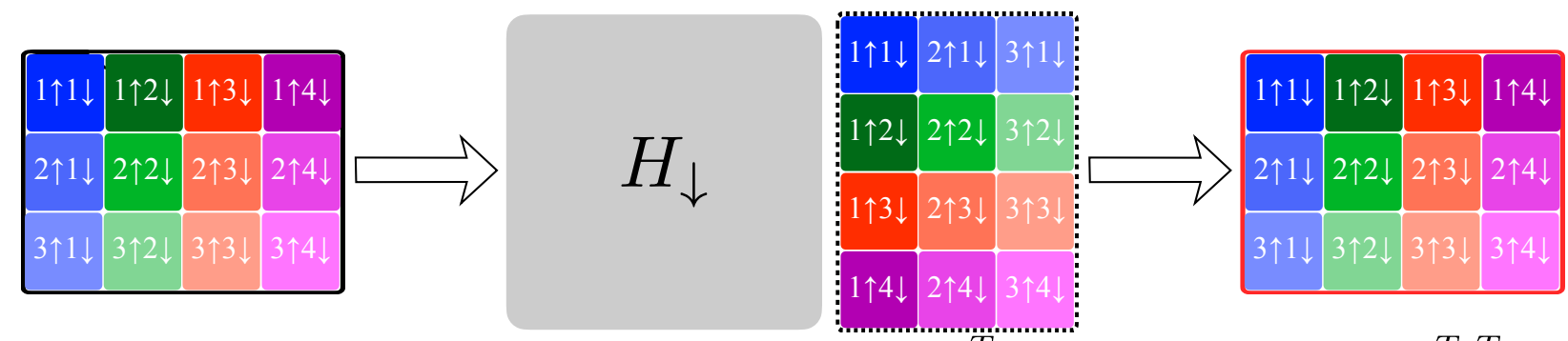

$|v\rangle \quad$ MPI_transpose

$|v\rangle^{T} \quad$ MPI_transpose

$\left[H_{\downarrow}|v\rangle^{T}\right]^{T}$

Figure 1: (Color online) Schematic representation of the MVP for the $\mathbb{Q}_{\uparrow} \otimes H_{\downarrow}$ term of the sector Hamiltonian. In this example the sector dimensions are $D_{\mathcal{S}}=D_{\uparrow} \times D_{\downarrow}=3 \times 4$. We assume to have $\mathrm{p}=4$ processors. The components of the vector are indicated by their index $v_{i_{\uparrow} i_{\downarrow}} \rightarrow i_{\uparrow} i_{\downarrow}$. Each column of the initial vector $|v\rangle$ corresponds to a different $\downarrow$ configuration, indicated by different colors. The shades of the same color correspond to different $\uparrow$ configurations. The columns are distributed to the processors. The distributed vector is parallel transposed so that each column corresponds to a different $\uparrow$ configuration and belongs to one processor. The MVP $|w\rangle^{T}=H_{\downarrow}|v\rangle^{T}$ can then be performed locally in the memory. The result $|w\rangle$ is obtained transposing back the resulting vector into the original format.

a matrix whose columns correspond to a set of spin- $\uparrow$ components for a given fixed spin- $\downarrow$ configuration:

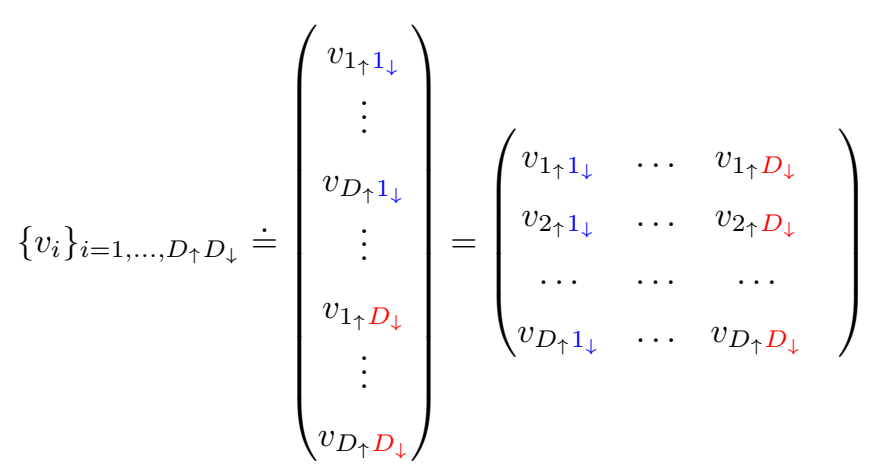

Each vector is distributed across the MPI processes such that a number $Q_{\downarrow}=D_{\downarrow} / \mathrm{p}$ of columns is assigned to each core. If present, the rest $R_{\downarrow}=\bmod \left(D_{\downarrow}, \mathrm{p}\right)$ is reassigned to the first $\mathrm{R}_{\downarrow}$ processes. This partitioning corresponds to distribute $\mathbf{Q}=D_{\uparrow} \times \mathrm{Q}_{\downarrow}$ vector elements to each process. Each term in (10) contributing to the MVP is evaluated separately.

\subsubsection{The $H_{d}$ term}

The diagonal term $H_{d}$ of the Hamiltonian is distributed among processes assigning Q rows, i.e. elements, to each core. Notably, the multiplication $H_{d}|v\rangle$ takes place locally in the memory on each process, i.e. without further MPI communication.

\subsubsection{The $H_{\uparrow} \otimes \rrbracket_{\downarrow}$ term}

The contribution of the term $H_{\uparrow} \otimes \mathbb{q}_{\downarrow}$ to the MVP involves only $\uparrow$-electrons elements, for any fixed configuration of the spin- $\downarrow$. In the MVP, this corresponds to run along the elements of each column of the matrix (12). The proposed MPI decomposition ensures that these elements are stored contiguously in the memory of each process. As such, like the diagonal term $H_{d}$, this term of the MVP can be computed locally in the memory of any processor, provided the (small) Hamiltonian $H_{\uparrow}$ is known to each process or it is evaluated on-the-fly.

\subsubsection{The $\rrbracket_{\uparrow} \otimes H_{\downarrow}$ term}

The product with the term $\square_{\uparrow} \otimes H_{\downarrow}$ involves $\downarrow$-electrons elements, for any fixed spin- $\uparrow$ configuration. In the MVP this corresponds to run along the rows of the matrix (12), ultimately leading to a highly non-local 
access to the memory. In a serial implementation this introduces only a minor performance degradation due to irregular cache access. However, in a parallel algorithm such non-locality represents a serious bottleneck, which requires massive MPI communication to transfer columns among different processes. A solution to such problem is to exchange the $\uparrow$ - and $\downarrow$-configuration indices $\left[\vec{i}_{\uparrow}, \vec{i}_{\downarrow}\right] \rightarrow\left[\overrightarrow{i_{\downarrow}}, \vec{i}_{\uparrow}\right]$, corresponding to an actual transposition of the matrix (12). The execution of transposed MVP recover locality in the processors memory.

The key issue, originally pointed out in Ref. 27, is reduced to perform a collective transposition of a data set, which in turn requires a suitable all-to-all communication. To this purpose, the MPI library makes available a parallel procedure, MPI_AlltoAllv, which transfers data such that the j-block, sent from the process $i$, is received by process $j$ and placed as block $i$. The corresponding communication pattern is schematically depicted in Fig. 7. This parallel transposition involves the minimum amount of data transfer necessary to execute the MVP, removing the communicational congestion and unlocking optimal parallel scaling. Details of the implementation are given in Sec. 6.7.

Summarizing, the MVP of the $\downarrow$-electrons part of the Hamiltonian proceeds in three steps: i) the vector $|v\rangle$ is transposed using collective MPI communication; ii) the multiplication is performed locally on each process; iii) the resulting vector is transposed back and added up to the result, see Fig. 1.

\subsubsection{The $H_{n d}$ term}

The last contribution to the MVP is from the non-diagonal term $H_{n d}$. This matrix contains elements which can not be reduced to any favourable form to perform a parallel MVP. As such, the execution of the MVP for this term necessarily relies on the MPI_AllGatherV-algorithm, discussed above. To this end, the matrix $H_{n d}$ gets row-distributed among all processes assigning Q rows to each core. The inclusion of this term is expected to spoil to some extent the scalability of the parallel MVP algorithm.

\section{Electron-phonon coupling}

The quantum impurity model (1) can be extended to include electron-phonon coupling. The impurity Hamiltonian takes the form: $\hat{H}=\hat{H}_{e}+\hat{H}_{e-p h}+\hat{H}_{p h}$, where $H_{p h}=\omega_{0} b^{+} b, H_{e-p h}=\sum_{\alpha \sigma} g_{\alpha} d_{\alpha \sigma}^{+} d_{\alpha \sigma}\left(b+b^{+}\right)$. The presence of additional, bosonic, degrees of freedom introduces major changes to the ED algorithm. However, such modifications do not spoil the essential aspects of the parallel algorithms outlined above.

\subsection{Fock space}

The Fock space should be extended to include the presence of the phonons. In order to deal with the unbounded dimensions of the local Hilbert space of the phonons we introduce a cut-off $N_{p h}$ to the number of available phonons. The Fock space of a system of $M_{p h}$ phonons is $\mathcal{F}_{p h}=\bigoplus_{n=0}^{M_{p h}} S_{+} \mathcal{H}_{p h}^{\otimes n}$, where $\mathcal{H}_{p h}=\left\{|0\rangle,|1\rangle, \ldots,\left|N_{p h}\right\rangle\right\}$ is the local phonon Hilbert space and $S_{+}$the symmetrization operator. The Fock space is given by the tensor product of the electronic and phononic spaces: $\mathcal{F}=\mathcal{F}_{e} \otimes \mathcal{F}_{p h}$. In the following we reduce to the case of a single phonon mode $M_{p h}=1$, localized in real space at the impurity site. As such the dimension of the phonon Fock space is given by $D_{p h}=N_{p h}+1$.

The coupling to phonons does not break the electronic QNs conservation nor it adds novel symmetries. Thus, the Fock space factorizes into novel sectors given by the product of any electronic sector and the phonons Hilbert space. Each sector is then identified by the tuple $\left[\vec{N}_{\uparrow}, \vec{N}_{\downarrow}\right]$ and has dimensions $\operatorname{dim}\left(\mathcal{S}\left[\vec{N}_{\uparrow}, \vec{N}_{\downarrow}\right]\right)=D_{\uparrow} D_{\downarrow} D_{p h} \equiv D_{\mathcal{S}}$

\subsection{Basis states}

According to this construction, the Fock basis of the electron-phonon system is composed of product states of the form $|\vec{n}, p\rangle=\left|n_{1 \uparrow}, \ldots, n_{N_{s} \uparrow}, n_{1 \downarrow}, \ldots, n_{N_{s} \downarrow}\right\rangle|p\rangle$, with $p=0,1, \ldots, N_{p h}$. The electronic and phononic creation (annihilation) operators, respectively, $d_{a \sigma}^{+}, a_{a \sigma}^{+}$and $b^{+}\left(d_{a \sigma}, a_{a \sigma}^{+}\right.$and $\left.b\right)$ act separately on the electronic and phononic part of the states. This separation of the Fock basis ensures that all the observations concerning the electronic configurations hold unaltered. In particular, each state $|\vec{n}\rangle$ is repeated 
$D_{p h}$ times. Consequently, we identify each Fock state by means of an integer index and an extended tuple $I \rightarrow\left[I_{e}, I_{p h}\right]=\left[\vec{I}_{\uparrow}, \vec{I}_{\downarrow}, I_{p h}\right]$, where $I_{p h}=0, \ldots, N_{p h}$ and $I_{e}$ identify the electronic Fock state. The relation between the Fock state index $I$ and the tuple reads:

$$
I=I_{1}+\sum_{i=2}^{2 N_{u d}} I_{i} 2^{(i-1) N_{b i t}}+I_{p h} 2^{2 N_{s}}
$$

In complete analogy, we use of the map $\overrightarrow{\mathcal{M}}_{\mathcal{S}}$ to build a basis for sector $\mathcal{S}\left[\vec{N}_{\uparrow}, \vec{N}_{\downarrow}\right]$, such that $I \in \mathcal{S}=$ $\left[\vec{I}_{\uparrow}, \vec{I}_{\downarrow}, I_{p h}\right] \in \mathcal{S} \stackrel{\overrightarrow{\mathcal{M}}_{\mathcal{S}}}{\longrightarrow}\left[\vec{i}_{\uparrow}, \vec{i}_{\downarrow}, i_{p h}\right]=i$, where $i_{\alpha \sigma}=1, \ldots, D_{\alpha \sigma}$ and $i_{p h}=1, \ldots, D_{p h}$. The tuple $\left[\vec{i}_{\uparrow}, \vec{i}_{\downarrow}, i_{p h}\right]$ identifies a state $|i\rangle=\left|\vec{i}_{\uparrow}, \vec{i}_{\downarrow}\right\rangle\left|i_{p h}\right\rangle$ of the sector $\mathcal{S}$ through the relation:

$$
i=i_{1 \uparrow}+\sum_{\sigma} \sum_{\alpha=2}^{N_{u d}}\left(i_{\alpha \sigma}-1\right) \prod_{\beta=1}^{\alpha-1} D_{\beta \sigma}+\left(i_{p h}-1\right) D_{\uparrow} D_{\downarrow} .
$$

\subsection{Hamiltonian construction}

In each sector $\mathcal{S}$ the Hamiltonian matrix of the electron-phonon coupled system has the following expression:

$$
H_{\mathcal{S}}=\rrbracket^{p h} \otimes H_{\mathcal{S}}^{e}+H^{p h} \otimes \rrbracket^{e l}+H_{\mathrm{e}-\mathrm{ph}}^{p h} \otimes H_{\mathrm{e}-\mathrm{ph}}^{e}
$$

where $H_{\mathcal{S}}^{e}$ is the electronic sector Hamiltonian, $H^{p h}$ is the phonon Hamiltonian, $H_{\mathrm{e}-\mathrm{ph}}^{p h}$ and $H_{\mathrm{e}-\mathrm{ph}}^{e}$ are, respectively, the phononic and electronic parts of the electron-phonon coupling term. Because of the factorization of the basis states, the construction of the electronic part of the Hamiltonian proceed as outlined above. The $H^{p h}$ term is diagonal in the phonon basis and independent of the electronic configuration. The electron-phonon coupling term $H_{\mathrm{e}-\mathrm{ph}}^{p h} \otimes H_{\mathrm{e}-\mathrm{ph}}^{e}$ can be factorized. The electronic part is diagonal, as the component $H_{\mathrm{e}-\mathrm{ph}}^{e}$ is proportional to the electronic density operators. However, the phonon factor $H_{\mathrm{e}-\mathrm{ph}}^{p h}$, being linear in the phonon displacement operator $\hat{x}=\left(b^{+}+b\right)$, is off-diagonal in the phonon states.

$$
\begin{aligned}
\left\langle\vec{i}_{\uparrow} \vec{i}_{\downarrow}\left|\left\langle i_{p h}\left|H_{\mathcal{S}}\right| \vec{j}_{\uparrow} \vec{j}_{\downarrow}\right\rangle\right| j_{p h}\right\rangle= & \left\langle\vec{i}_{\uparrow} \vec{i}_{\downarrow}\left|H_{\mathcal{S}}^{e}\right| \vec{j}_{\uparrow} \vec{j}_{\downarrow}\right\rangle \delta_{i_{p h} j_{p h}}+ \\
& \delta_{\vec{i}_{\uparrow} \vec{j}_{\uparrow}} \delta_{\vec{i}_{\downarrow} \vec{j}_{\downarrow}}\left\langle i_{p h}\left|H^{p h}\right| i_{p h}\right\rangle+ \\
& \left\langle\vec{i}_{\uparrow} \vec{i}_{\downarrow}\left|H_{\mathrm{e}-\mathrm{ph}}^{e}\right| \vec{i}_{\uparrow} \vec{i}_{\downarrow}\right\rangle\left\langle i_{p h}\left|H_{\mathrm{e}-\mathrm{ph}}^{p h}\right| j_{p h}\right\rangle
\end{aligned}
$$

\subsection{Parallel matrix-vector product}

The factorization of the electrons and phonons configurations in the basis states allows to introduce only few changes to the MVP algorithm, designed to optimize the parallel execution. The most important step concerns the distribution of the vectors among the processes. To this end, we observe that the vector decomposition along the sector basis is such that the electronic configurations are "repeated" $D_{p h}$ number of times:

$$
|v\rangle=\sum_{i, i_{p h}} v_{i, i_{p h}}|i\rangle\left|i_{p h}\right\rangle=\sum_{i_{\uparrow}=1}^{D_{\uparrow}} \sum_{i_{\downarrow}=1}^{D_{\downarrow}} \sum_{i_{p h}=1}^{D_{p h}} v_{i_{\uparrow}, i_{\downarrow}, i_{p h}}\left|i_{\uparrow}\right\rangle\left|i_{\downarrow}\right\rangle\left|i_{p h}\right\rangle
$$

Thus, each vector is distributed among the MPI processes such that $Q_{\downarrow}^{p h} \times D_{p h}=D_{\downarrow} / \mathrm{p} \times D_{p h}$ columns, i.e. $D_{p h}$ copies of the $Q_{\downarrow}$ electronic configurations, are assigned to each core. Each process holds $\mathbf{Q}^{p h}=$ $D_{\uparrow} \times \mathbf{Q}_{\downarrow} \times D_{p h} \equiv D_{\uparrow} \times \mathbf{Q}_{\downarrow}^{p h}$ elements.

Then, for any fixed phonon configuration, the execution of the MVP for the purely electronic part of the Hamiltonian proceeds along the same lines. The product with the phonon Hamiltonian $H^{p h}$, diagonal in the electronic states, only involves iterations along the phonon index for all the electronic elements residing on each processor, i.e. it is local in the memory. Finally, according to the vector distribution among the 

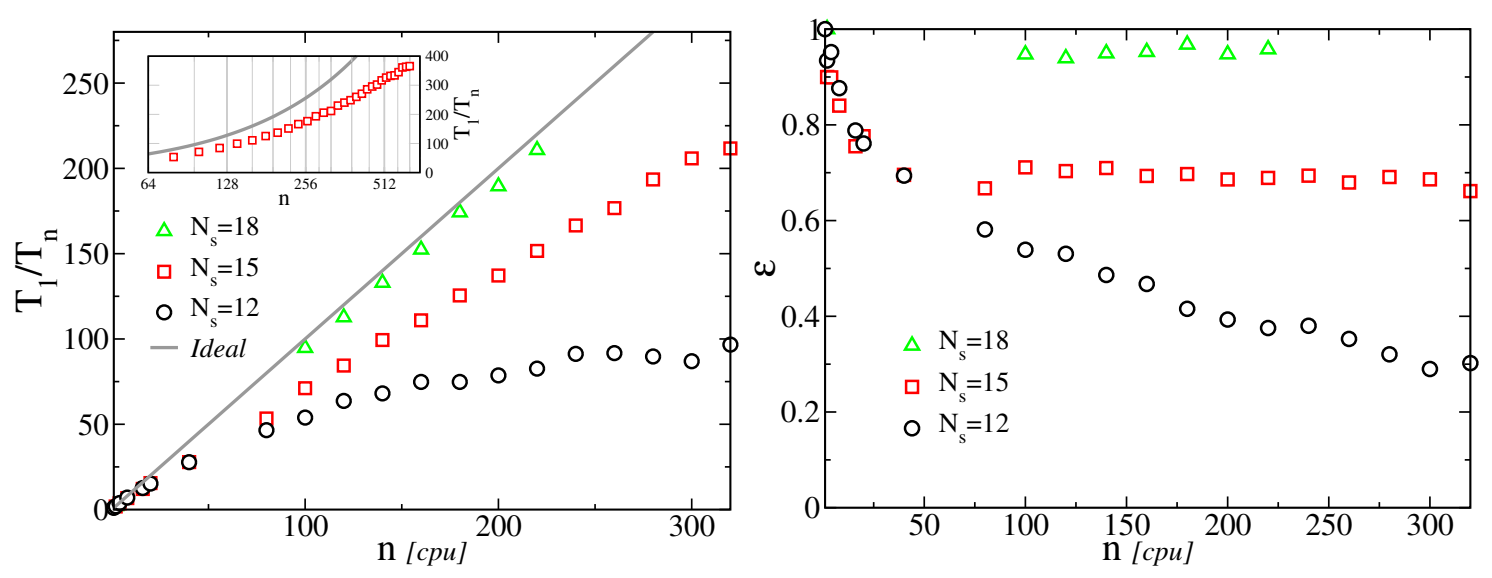

Figure 2: (Color online) Left: Parallel speed-up $T_{1} / T_{n}$ as a function of the CPUs number $n$. Data are for systems with increasing number of levels $N_{s}=12,15$ and 18. The ideal scaling (solid gray line) is reported for comparison. The parallel gain saturates at $n \simeq 256$ for $N_{s}=12$, it shows sub-ideal behavior for $N_{s}=15$ and nearly ideal scaling for $N_{s}=18$. Data for $N_{s}=18$ are normalized to $n=2$. Inset: Parallel speed-up as a function of CPUs number for $N_{s}=15$ in a wider range of $n$ and on a semi-log scale. The results shows the saturation of the parallel gain for $n>512$. Right: Parallel efficiency $\varepsilon=T_{1} / n T_{n}$ as a function of the CPUs number $n$. Data as in left panel.

processors, the product with the electron-phonon Hamiltonian is straightforward. The $H_{\mathrm{e}-\mathrm{ph}}^{e}$ Hamiltonian is diagonal and distributed to the processors in shares of size $Q$ rows, so its contribution is local in the memory. The term $H_{\mathrm{e}-\mathrm{ph}}^{p h}$, known to each process, connects Q columns of electronic elements having different phonon index, yet residing in the memory of the same process and requiring no further change to the MVP algorithm.

\section{Benchmarks}

We present some benchmark results for the massively parallel ED algorithm outlined above. The calculations have been performed on HPC cluster, using Intel Xeon E5-2680 v2 processors with 2 sockets, 10 cores, 2 threads per core and 40 GB RAM. We considered a multi-orbital quantum impurity model with a total of $N_{s}$ electronic levels. The impurity hosts $N_{\alpha}$ orbitals, each coupled via an hopping amplitude to $N_{b}$ bath levels, with random energies in the interval $[-2 D, 2 D]$, with $D=1$ setting the energy unit. The tests have been executed using total spin $\uparrow, \downarrow$ electrons occupation QNs in the half-filling sector $N_{\uparrow}=N_{\downarrow}$. This setup corresponds to the largest possible sector dimension, which is ideal to test scaling properties of the algorithm. Timing is relative to the evaluation of the lowest state of the spectrum, using Lanczos or P-Arpack ${ }^{45}$ algorithm with on-the-fly evaluation of the MVP, i.e. without storage of the Hamiltonian. In order to reduce the intrinsic errors, we performed repeated calculations for different realizations of the bath energy distribution. The data presented in this section are averaged over 10 realizations.

The speed-up of the parallel algorithm and its efficiency are reported in Fig. 2 for an increasing number of total electronic levels $N_{s}$. For a system with $N_{s}=12$, the parallel gain rapidly saturates for $n \simeq 250$ CPUs. Indeed, for such small system the ratio between calculation and communication time quickly becomes unfavourable, leading to a premature saturation of the performances. This is further underlined by the decreasing behavior of the parallel efficiency $\varepsilon$ reported in the right panel. The scaling improves for larger systems. In particular, we observed a sub-linear scaling for systems of $N_{s}=15$, which displays a saturation tendency only for the largest accessible number of processes (see inset). For this size, the parallel efficiency shows a nearly constant behavior up to the largest number of processor. Finally, for the largest system with $N_{s}=18$ we observed a quasi ideal scaling and efficiency near one in the entire range of CPU number analyzed.

In order to test the solidity of the scaling with respect to the intrinsic properties of the Lanczos algorithm, we compared the single vector Lanczos implementation with respect to a fully fledged P-Arpack algorithm ${ }^{45}$. 

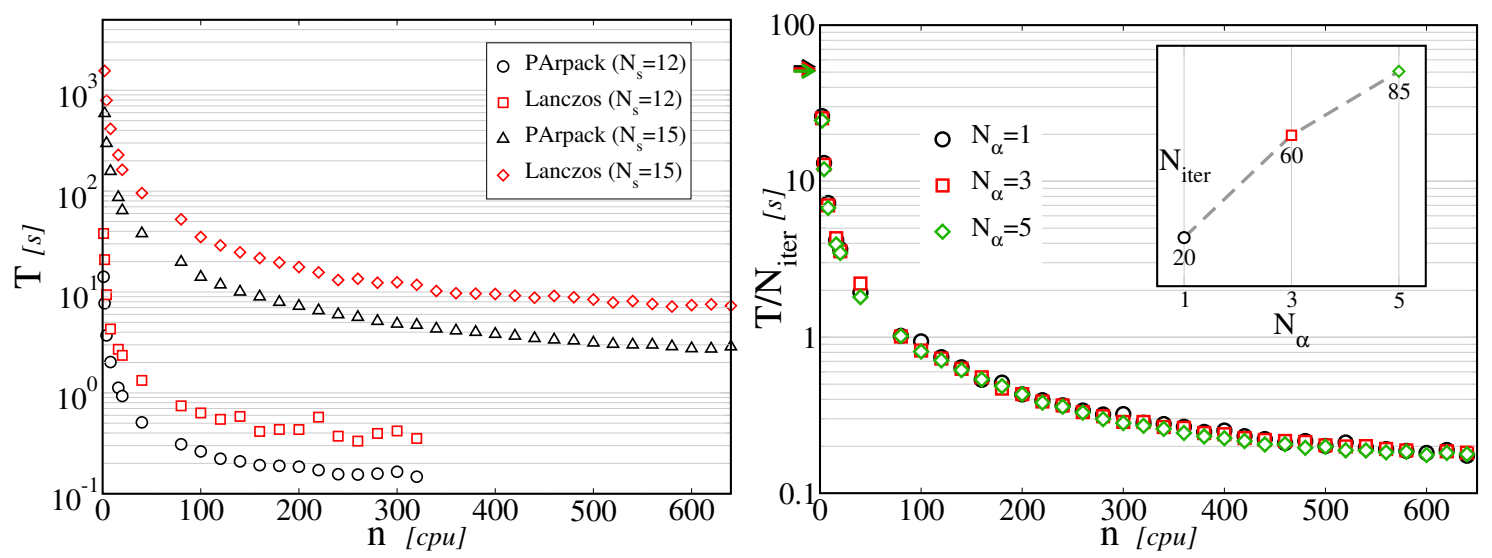

Figure 3: (Color online) Left panel: Total execution time $T$ as a function of the number of CPUs $n$, for systems of $N_{s}=12$, 15 levels. Data compare results obtained with P-Arpack (black circles and triangles) against simple Lanczos (red squares and diamonds) methods. Right panel: Average execution time for a single Lanczos iteration $T / N_{i t e r}$ as a function of CPUs number $n$. Data are for a system of $N_{s}=15$ levels with a number of orbitals $N_{\alpha}=1,3,5$. The data show that single Lanczos iteration time is essentially independent of the orbitals number $N_{\alpha}$. Inset: number of Lanczos iterations $N_{\text {iter }}$ for a single groundstate calculation as a function of orbitals number $N_{\alpha}$. Data show that the iterations number linearly increases with $N_{\alpha}$ and so does the total execution time $T$.

For the P-Arpack calculations we used a block of ncv $=10$ vectors, which we determined to optimize the execution of our program. The memory footprint as well as the number of matrix-vector operations among the two methods are very different, potentially leading to dissimilar scaling behavior. The results in the left panel of Fig. 3 reveal that the scaling of the two methods is indeed similar, i.e. the same order of magnitude. Interestingly however, despite its higher complexity, the more optimized P-Arpack algorithm performed consistently better than the plain Lanczos method.

A crucial aspect towards application of the algorithm is the scaling behaviour with respect to the number of orbitals. The multi-orbital interaction includes terms which are not diagonal in the electronic configurations, such as spin-flip and pair-hopping. These terms have a possible detrimental impact on the parallel scaling, thus we temporarily exclude their contributions and we will analyze their influence separately. In multi-orbital systems the hopping matrices include more terms with respect to the single orbital, due to the presence of several hopping channels among orbitals. In order to characterize quantitatively these aspects we studied the scaling of the algorithm as a function of an increasing number of orbitals $N_{\alpha}$, see the right panel of Fig. 3. Our results show that the single Lanczos iteration time is essentially independent of the number of orbitals. However, the number of iterations required to determine the lowest eigenstate increases linearly with the number of orbitals, see inset. We conclude that, although the resulting total time has an excellent sub-linear scaling with the number of processes, it linearly depends on the number of orbitals, i.e. the more the orbitals the larger the solution time for a fixed number of processes.

As mentioned above, we analyzed the effect of non-diagonal interaction terms on the scaling behavior of our algorithm. The pair-hopping and spin-exchange terms cannot be reduced to hopping events involving a single spin orientation. As such we can not take advantage of the special properties of the basis states to perform the parallel MVP, possibly spoiling the nearly optimal scaling observed for the case of densitydensity interaction only. In order to quantify this effect in Fig. 4 we compare the speed-up behavior of the density-density and full Kanamori interaction, in two systems of 3 and 5 orbitals. As before, the densitydensity interaction has a sub-linear scaling, which saturates around $n>600$ processes. The behavior of the full Kanamori case is however very different. An initial good speed-up obtained with few tens of processes, quickly saturates to a value of about 50 for any number of CPU $n>100$. In fact, above this threshold the burden of the MPI communication needed to reconstruct the whole vector and perform the product for $H_{n d}$ becomes predominant and prevents further improvement of the scaling.

The scaling properties of the parallel algorithm are mostly influenced by the dimensions of the sector 

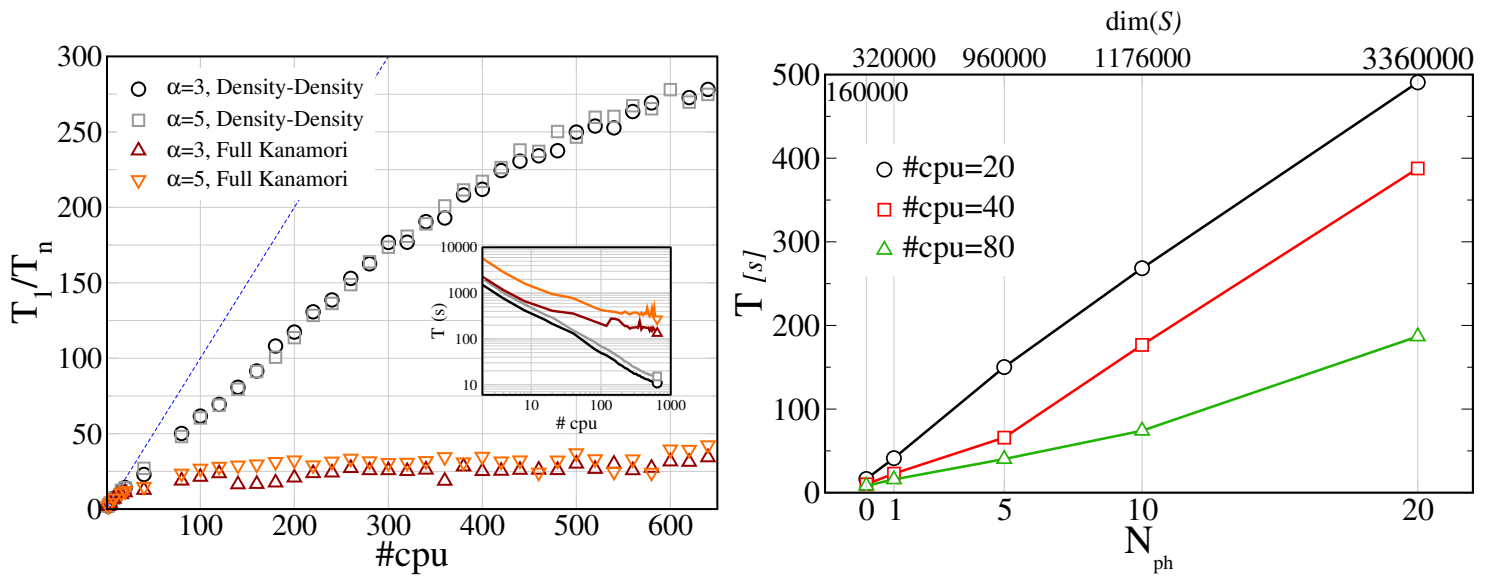

Figure 4: (Color online) Left panel: Parallel speedup of the ED algorithm as a function of the number of processes for a single groundstate calculation. Data are obtained using P-Arpack $(n c v=10)$, for a number of orbitals $N_{\alpha}=3,5, N_{s}=15$ and two type of interaction terms: Ising or density-density (black circles and gray squares) and full Kanamori including spin-flipping and pair-exchange (red up triangles and orange down triangles). Ideal scaling (dotted blue line) is reported for comparison. The data show that inclusion of non-diagonal Hamiltonian terms $H_{n d}$ quickly saturates the scaling. Right panel: Total execution time for the groundstate calculation as a function of the number of phonons $N_{p h}$ (bottom axis) or sector dimension (top axis). Data are obtained using P-Arpack $(n c v=10)$, for $N_{s}=12$ in the half filled sector $N_{\uparrow}=N_{\downarrow}=6$ for total occupation per spin QNs. The top $\mathrm{x}$-axis shows the dimensions of the $\operatorname{sector} \operatorname{dim}(\mathcal{S})$ for a given number of phonons. $N_{p h}=0$ corresponds to the purely electronic problem.

associated to a specified set of QNs as well as the sparseness of the hopping Hamiltonian matrices, which is partially controlled by the number of orbitals. Thus, the scaling for the case of orbital resolved QNs is qualitatively similar to the results discussed above, provided the sector dimensions are equal, a condition that is fulfilled only for larger value of $N_{s}$.

Finally, we discuss the influence on the scaling of the electron-phonon coupling. The presence of the phonons leads to a linear in $N_{p h}$ increase of the total sector dimension, see right panel in Fig. 4 . As the overall cost of the ED solution is proportional to such dimension, the inclusion of phonons limits the number of electronic degrees of freedom available to describe the impurity problem. For instance, with a small number of phonons $N_{p h} \simeq 10$, systems with up to three orbitals $N_{\alpha}=3$ can still be efficiently solved. Such an increase in the dimension of the sector caused by electron-phonon coupling influences the scaling of the parallel MVP algorithm, leading at most to a linear increase of the time. Indeed, the product with the electronic part of the Hamiltonian, i.e. the most time consuming part of the MVP algorithm, should be repeated for each phonon configuration ultimately reducing by a suitable factor the overall execution performances. This expectation is confirmed by the results reported in the right panel of Fig. 4, where we present the scaling of the ED algorithm with respect to the number of phonons $N_{p h}$ for a system with $N_{s}=12$ in the half-filling sector $N_{\uparrow}=N_{\downarrow}$ and total occupation per spin QNs. The results show that the total execution time for the groundstate calculation scales linearly, with a slope that depends on the CPU number.

\section{Library description and implementation}

The structure of the library is schematically represented in Fig. 5, using a simplified Unified Modeling Language (UML) diagram. The entire library is written in Fortran using object oriented programming within a modules structure. The Fortran interface is provided by the top-end module exposing to the end-user the procedures required to setup and solve a quantum impurity problem. The Python API are provided by a pure Python module edipy. In what follows we describe in more details the structure and the implementation of the most relevant parts of the EDIpack library. In order to unify the notation we 


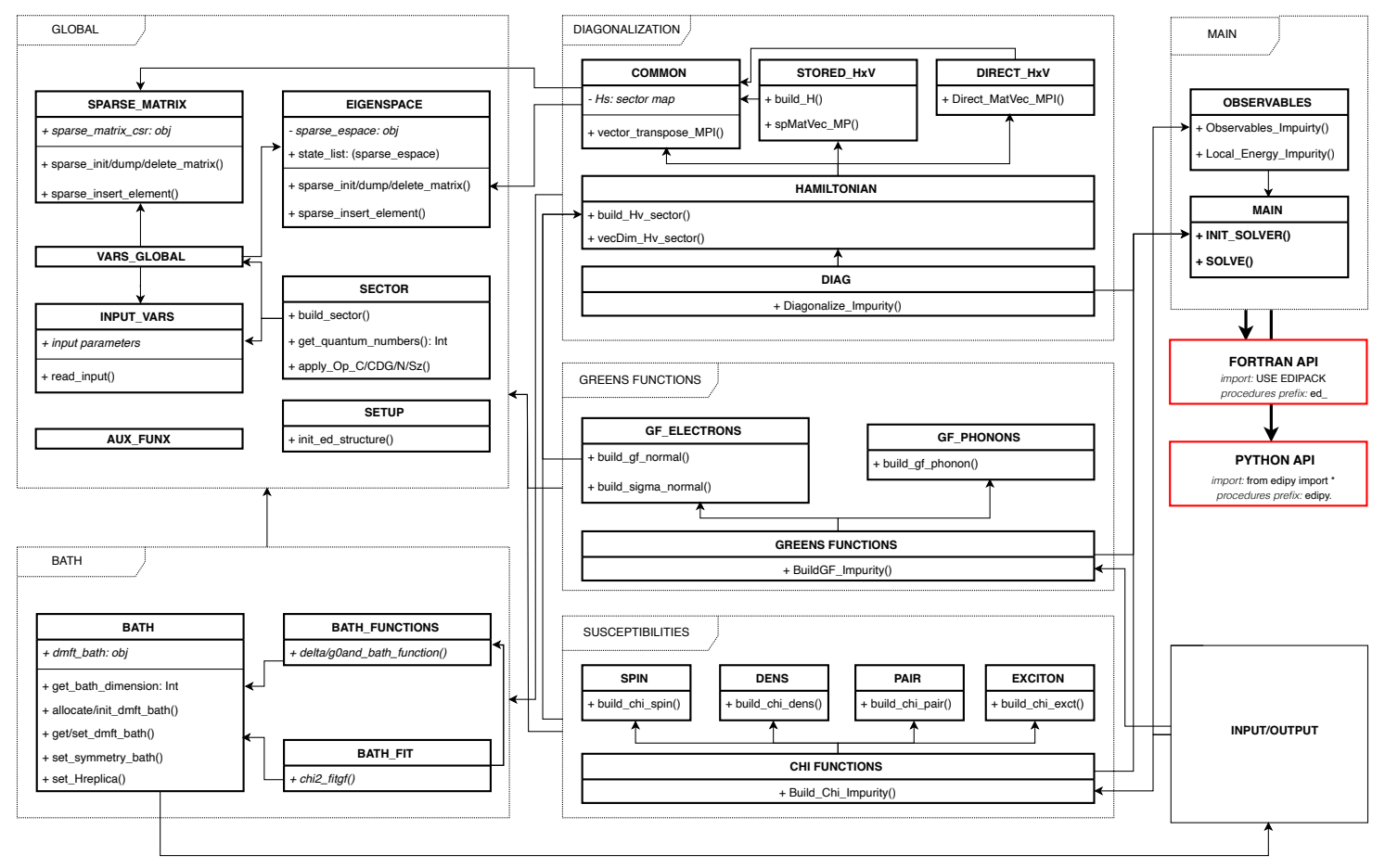

Figure 5: (Color online) Simplified UML diagram of the EDIpack library. The library is divided in several abstract work packages Global, Bath, Diagonalization, GreensFunctions, Susceptibilities, Input/Output, Main aimed to carry out specific tasks or to contain global objects or procedures. General classes and setup procedures are defined in the Global package. Discretized bath is handled in the Bath package. The hamiltonian matrix is diagonalized in the Diagonalization package and its results are used in GreensFunctions, Susceptibilities to construct dynamical correlations functions. The Fortran API, i.e. the library interfaces, is provided by the top end module EDIpack, providing access to a selected number of global procedures. The Python binding edipy is built using the numpy.f2py tool on a Fortran layer in combination with a pure Python module. 
henceforth indicate with the generic prefix ?=ed_ or edipy. either Fortran or Python procedures.

\subsection{Sparse matrix class}

A sparse matrix storage is performed using a dedicated custom class, contained in the SPARSE_MATRIX module. The class defines a sparse_matrix_csr object as a simplified hash-table. The keys corresponds to the rows of the matrix while the value is associated to a pair dynamical arrays, containing values and columns location of the non-zero elements of the sparse matrix. The sparse_matrix_csr object can be stored either serially, i.e. one copy per process, or be parallel distributed assigning a number of keys/values to each process. The elements are progressively stored in the dynamic arrays using sp_insert_element procedure, ultimately making use of the Fortran intrinsic move_alloc. This ensures a faster execution compared to implicit reallocation, i.e. $v e c=[\mathrm{vec}$, new_element]. This solution enables to deal with the a priori unknown number of non-zero elements on each row, to optimize the memory footprint and to guarantee $O(1)$ access to any element of the matrix, which are crucial aspect to speed-up the execution of the MVP.

\subsection{Eigenspace class}

The storage of the eigenvalues and eigenvectors is a key aspect of the ED algorithm. In our implementation this task makes use of a dedicated class, defined in EIGENSPACE. The class contains the object sparse_espace: an ordered single linked list storing the eigenvalue (the sorting key), the eigenvector and the corresponding QNs. In parallel mode the eigenvectors are automatically distributed to all processors in shares of size Q (see Sec. 3) and can be accessed only through pointer functions to avoid memory duplication.

For zero temperature calculations only the groundstates are stored in the list. For a finite temperature also excited states are saved. In order to avoid unbounded growth of the list we adopt an annealing truncation mechanism. In the first call we collect a number lanc_nstates_sector of states from each sector, up to a given maximum number lanc_nstates_total. Both parameters are initially set by the user from the input file. The list is truncated to keep the states which fulfil the condition $e^{-\beta\left(E_{i}-E_{0}\right)}<$ cutoff, where $E_{i}$ is the energy of the $i^{\text {th }}$ state in the list, $E_{0}$ is the groundstate energy, $\beta=1 / T$ is the inverse temperature $\left(k_{B}=1\right)$ and cutoff is an input parameter fixing an a priori energy threshold. Annealing is achieved by successive diagonalization of the problem. The numbers of states required to any sector $\mathcal{S}$ contributing to the list is increased by lanc_nstates_step or it is reduced otherwise. After few calls (of the order of ten) the distribution among the sectors of the numbers of states reaches a steady state. The corresponding annealed list contains all and just the states contributing to the spectrum up to the required energy threshold. A histogram of the number of states for each sector is produced after each diagonalization to check the evolution of their distribution.

\subsection{Sector construction}

The setup of the Fock space sectors is performed in the module SECTOR. The build_sector procedure is used to construct any given sector $\mathcal{S}\left[\vec{N}_{\uparrow}, \vec{N}_{\downarrow}\right]$ by setting up the map $\overrightarrow{\mathcal{M}}$. Operationally the map corresponds to a suitable structure which holds $2 N_{u d}$ integer arrays. Any such array has a length $D_{\alpha \sigma}$, with $\alpha=1, \ldots, N_{u d}$, $\sigma=\uparrow, \downarrow$. The arrays are constructed iteratively by looping over the integers $I_{\alpha \sigma}=0, \ldots, 2^{N_{b i t}}-1$. The state index is appended into the array if its bit decomposition corresponds to the required QNs value. The key part of build_sector is shown below:

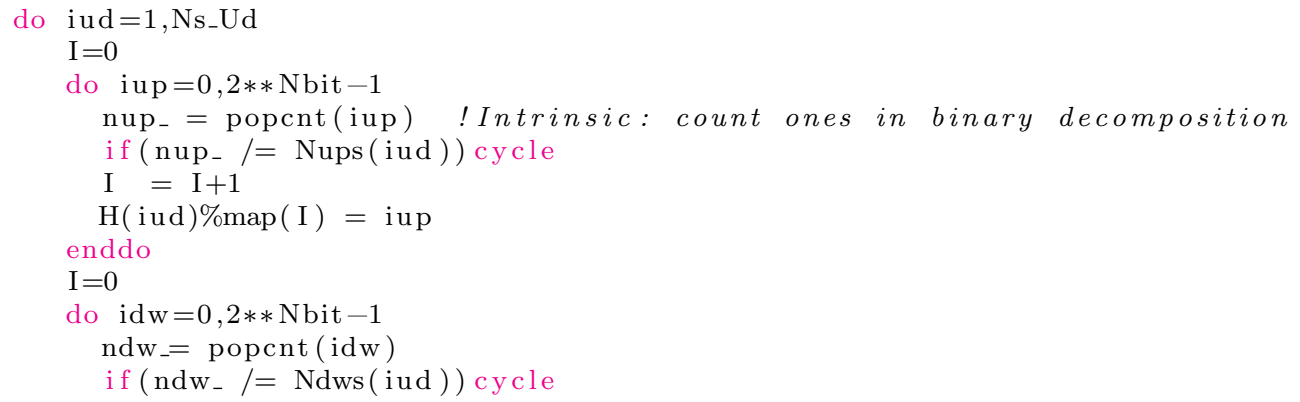



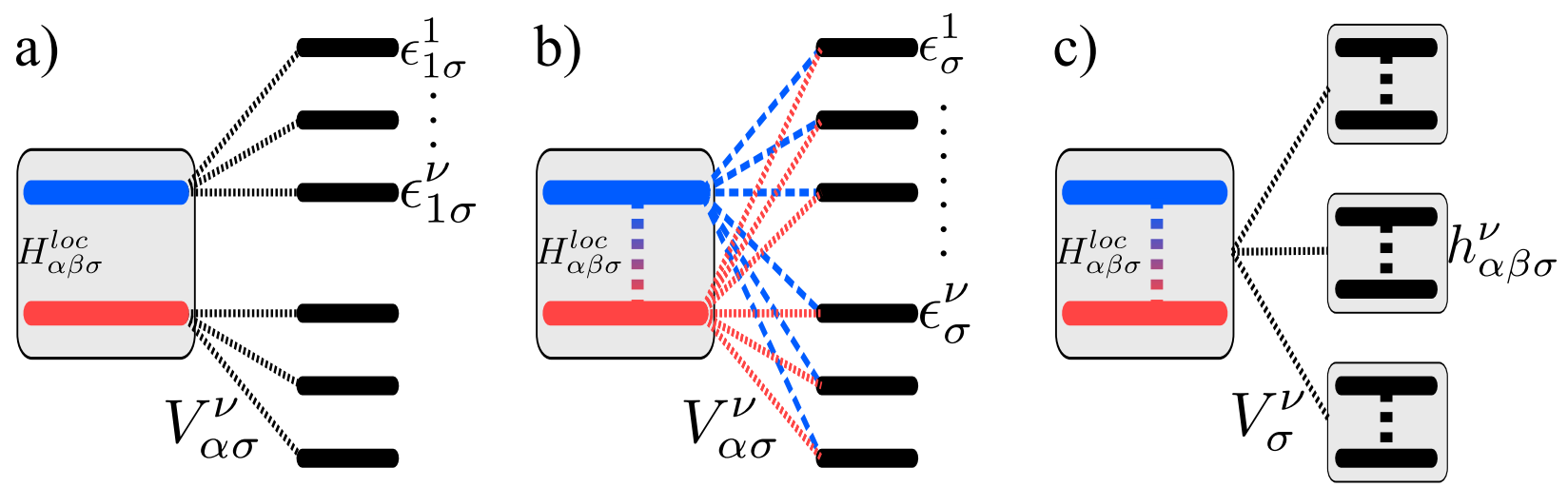

Figure 6: (Color online) Schematic illustration of the three available bath topologies: normal (a), hybrid (b) and replica (c). (a) In the normal topology a set of $N_{b}$ electronic levels is indipendently coupled to each impurity orbital. (b) In the hybrid bath a set of $N_{b}$ of electronic levels is coupled via different amplitude to each impurity orbital. (c) In the replica bath each electronic level reproduces the structure of the impurity and is coupled via a single amplitude.

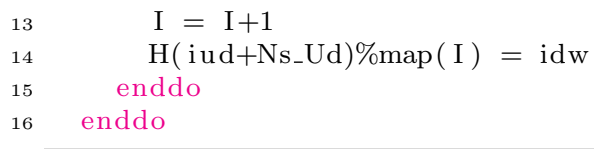

Inversion of such maps is achieved through a binary search reverse-lookup algorithm. The small performance loss in access time of order $O(N \log (N))$ is overly compensated by the lower memory footprint.

\subsection{Bath class}

Depending on the symmetries of the quantum impurity problem different topologies of the non-interacting bath can be devised. In the EDIpack library we include three general bath topologies. The three cases are selected by the input parameter bath_type=normal, hybrid, replica ${ }^{46}$. A schematic description of the three cases is reported in Fig. 6.

In the first case, i.e. normal, $N_{b}$ bath levels are directly coupled to each orbital. The total number of electronic levels is then $N_{s}=\operatorname{Norb}(\operatorname{Nbath}+1)$. Each bath level $\nu$ corresponding to the impurity orbital $\alpha$ bears a local energy $h_{\alpha \beta \sigma}^{\nu}=\epsilon_{\alpha \sigma}^{\nu} \delta_{\alpha \beta}$ and is coupled to the impurity by an amplitude $V_{\alpha \sigma}^{\nu}$. This bath topology can be used whenever $H_{\alpha \beta \sigma}^{l o c}$ is diagonal in the orbital indices and no particular orbital symmetry breaking are expected. Should these conditions not be verified another bath topology must be considered.

In the hybrid case, the $N_{b}$ bath levels are all coupled to the different impurity orbitals, see Fig. 6b. In this case the total number of electronic levels is $N_{s}=$ Norb + Nbath. The bath levels carry a local energy $h_{\alpha \beta \sigma}^{\nu}=\epsilon_{\sigma}^{\nu}$, i.e. independent of the orbitals indices, while they hybridize with each impurity orbital with amplitude $V_{\alpha \sigma}^{\nu}$. In this configuration, the bath can describe any effect associated to inter-orbital processes, at the cost of introducing possibly redundant bath parameters.

A way to limit the redundancy of the bath parameters for multi-orbital quantum impurity problems with off-diagonal terms is to consider the replica bath, see Fig. 6c. In this case the total number of electronic levels is $N_{s}=\operatorname{Norb}($ Nbath +1$)$. In this topology each bath level corresponds to a replica of the impurity, i.e. it has the same local structure of the impurity site, with parameters to be determined and encoded in the Hamiltonian $h_{\alpha \beta \sigma}^{\nu}$. Each replica couples to the impurity via a single amplitude $V_{\sigma}^{\nu}$. The choice of the structure for $h_{\alpha \beta \sigma}^{\nu}$ is a key step to exploit the nature of this topology. To this end, in EDIpack we assumed that each bath Hamiltonian can be linearly decomposed as

$$
h_{\alpha \beta \sigma}^{\nu}=\sum_{p=1}^{N_{p}} \lambda_{\sigma}^{\nu}(p) \Gamma_{\alpha \beta}(p)
$$


where the $N_{p}$ matrices $\Gamma_{\alpha \beta}(p)$ form a suitable basis for the local Hamiltonian and $\lambda_{\sigma}^{\nu}(p) \in \mathbb{R}$ are variational parameters for each bath level, i.e. replica. The setup of the matrix basis $\Gamma_{\alpha \beta}(p)$ and the initial values of the variational parameters is possible using the interface procedure ?set_Hreplica. This procedure accepts two type of inputs. The first is the local part of the impurity Hamiltonian $H^{l o c}$, as an array with rank-2 or rank-4 (see Table 2). In this case all the non-zero elements of the $H^{l o c}$ are identified, their values are used to initialize the paramaters $\lambda_{\sigma}^{\nu}(p)$. For any such non-zero element a matrix $\Gamma(p)$ with the same dimension of $H^{l o c}$ is defined containing a one only at the position of such element. The set of matrices $\Gamma(p)$ naturally decomposes the local non-interacting impurity Hamiltonian $H^{l o c}$. The second input method requires the user to directly pass the set of matrices $\Gamma(p)$, as rank- 5 array and the list of initial values for the corresponding variational parameters, see the table 2. Although potentially less generic, this method allows the user to carefully select the basis matrices in order to reflect specific physical aspects, e.g. the existence of possible symmetry breaking.

The bath class is handled using a reverse communication strategy. Within the library the bath is described by the class dmft_bath. On the user side the bath parameters are stored in a rank-1 array of real numbers, which provides the main input of the solver. The correct dimensions of such array are retrieved using ?get_bath_dimension procedure. Within the BATH class we also provides methods to operate specific symmetry trasformation onto the bath, e.g. particle-hole, spin symmetrization, etc. More general operations can be constructed by the user through suitable methods which allows to get and set specific components of the bath.

\subsection{Bath fit}

In the DMFT context the bath is updated using a suitable optimization of the bath parameters against a given realization of the Weiss field $\mathcal{G}_{0}^{-1}{ }_{\alpha \beta}(z)$, or the corresponding Delta function $\Delta_{\alpha \beta}(z)=(z+\mu) \delta_{\alpha \beta}-$ $H_{\alpha \beta}^{l o c}-\mathcal{G}_{0}^{-1}{ }_{\alpha \beta}(z), z \in \mathbb{C}$, determined by the self-consistency procedure ${ }^{4}$. This step corresponds to a projection of the Weiss field (or the Delta function) onto the space of the Anderson quantum impurity model functions $\mathcal{G}_{0}^{A n d}(z)=\left[(z+\mu) \delta_{\alpha \beta}-H_{\alpha \beta}^{l o c}-\Delta_{\alpha \beta}(z)\right]^{-1}$, with $\Delta_{\alpha \beta}^{A n d}(z)=\sum_{\nu} V_{\alpha}^{\nu}\left(z-h_{\alpha \beta}^{\nu}\right)^{-1} V_{\beta}^{\nu}$, spanned by the variational paramaters $\{V, h\}$.

This optimization can be performed using different algorithms ${ }^{23,47}$. In EDIpack we offer to perform this task using a conjugate gradient (CG) minimization of the cost function:

$$
\chi=\sum_{n=1}^{L_{f i t}} \frac{1}{W_{n}} \sum_{\alpha \beta}\left|X_{\alpha \beta}\left(i \omega_{n}\right)-X_{\alpha \beta}^{A n d}\left(i \omega_{n} ;\{V, h\}\right)\right|^{q}
$$

where $X_{\alpha \beta}=\mathcal{G}_{0 \alpha \beta}, \Delta_{\alpha \beta}$ are the user supplied local functions and $X_{\alpha \beta}^{A n d}=\mathcal{G}_{0}{ }_{\alpha \beta}^{\text {And }}, \Delta_{\alpha \beta}^{\text {And }}$ the Anderson impurity model function, defined above. The fit procedures are contains in the module BATH_FIT. In order to exploit regularity of the functions the fit is performed using imaginary Matsubara frequency. The actual form of $X_{\alpha \beta}$ is controlled by the input parameter cg_Scheme=Weiss, Delta.

Given the importance of the fit for the convergence of DMFT calculations, in EDIpack we introduced different specific control parameters. This aims to give the user the possibility of fine tuning the fit and adapt its behavior to specific situations. Two distinct CG algorithms can be used in the library, as controlled by the input variable $\mathrm{cg}$ method=0,1. The value 0 corresponds to a Fletcher-Reeves-Polak-Ribiere minimisation algorithm, adapted from Numerical Recipes ${ }^{48}$. In order to guarantee back-compatibility with respect to the literature, we also provide the possibility of using the minimization procedure published in Ref. 4 which has been largely used in the DMFT community.

The gradient $\nabla \chi$ required by the CG algorithm can be evaluated either analytically or numerically as controlled by the value of the input parameter cg_grad=0,1. For cg_method=1 only numerical gradient can be used. The number of Matsubara frequency $L_{f i t}$ used in the fit procedure is controlled by the input parameter cg_Lfit. This parameter can be used to restrict the fit to the low frequency part as the large frequency tails of the local Matsubara functions have universal behavior. Similarly, the weight $W_{n}=1,1 / L_{f i t}, 1 / \omega_{n}$ is used to enhance the weight of low energy part with respect to the intermediate-to-large energy one in the cost function. This is controlled by the input parameter cg_Weight=0,1,2. Another factor contributing to 


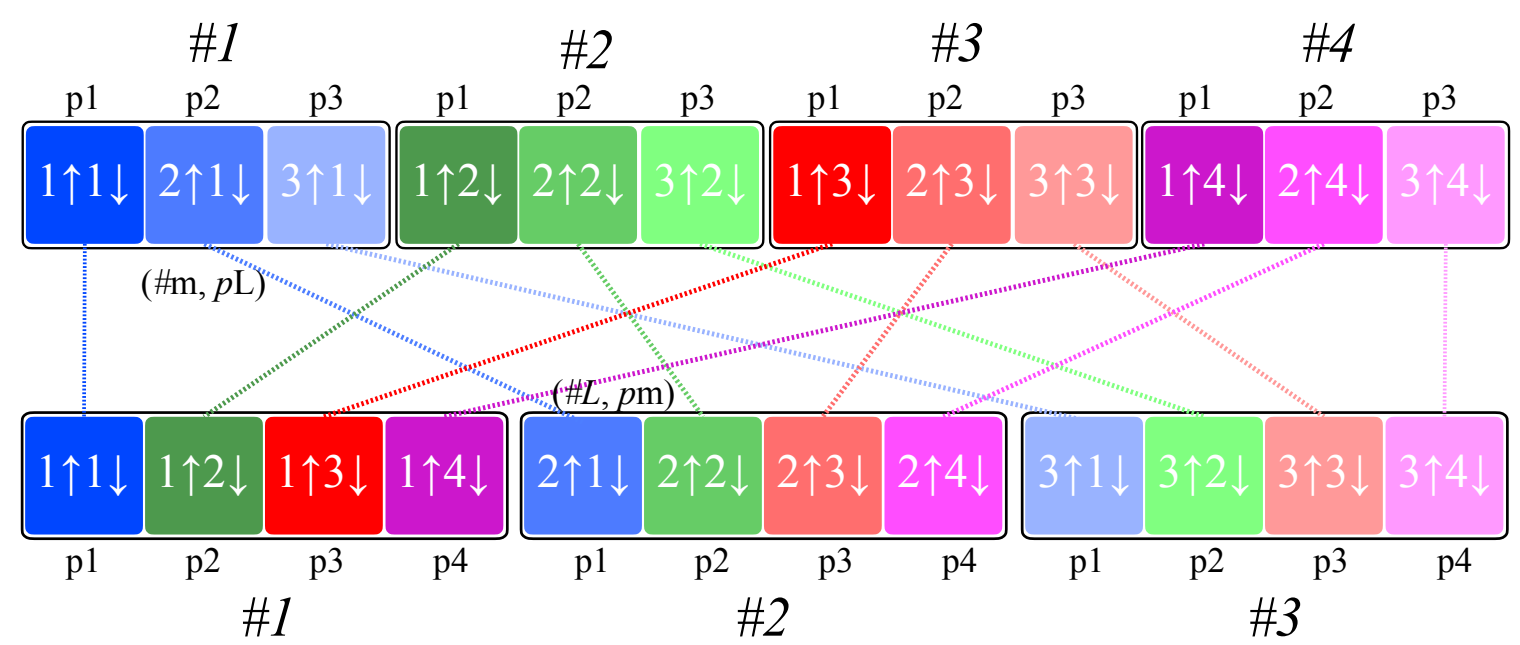

Figure 7: (Color online) Schematic representation of the MPI_AllToAllV parallel transposition of a vector. We consider the same case as in Fig. 1, i.e. vector dimensions $D_{\mathcal{S}}=D_{\uparrow} \times D_{\downarrow}=3 \times 4$ and $\mathrm{p}=4$ processors. The initial vector (top part) is distributed so that each processor, indicated with $\# \mathrm{~m}$ and $\mathrm{m}=1, \ldots, 4$, holds one column, corresponding to a different color. Each column contains 3 data components, indicated with $p \mathrm{~L}, \mathrm{~L}=1,2,3$ and corresponding to different shades of the same color. Each vector component is identified by the pair $(\# \mathrm{~m}, p \mathrm{~L})$. The procedure MPI_AllToAllV sends the element at L of the processor $\mathrm{m}$ to the processor $\mathrm{L}$ placing it at position $\mathrm{m}$, i.e. each component $(\# \mathrm{~m}, p \mathrm{~L})$ is moved to (\#L, $p \mathrm{~m})$. The final result (bottom part) is the transposed vector. Note that processor 4 does not receive any component although it participates in the communication process.

determine the behavior of the fit is the power $q$ of the cost function $\chi$. By tuning this parameter, controlled by input variable cg_pow, it is possible to enhance or suppress the differences in the $\chi$ in order to improve (or simplify) the optimization procedure.

Finally, we introduced few additional parameters which aim to control the quality of the fit. The first is the fit tolerance, controlled by the input parameter cg_Ftol, which determines the convergence threshold of the minimization procedure. The second is the maximum number of iterations cg_Niter allowed for the CG algorithm to determine the minimum. The last parameter cg_stop=0,1,2 selects the exit condition of the minimization procedure. We envisaged three possible conditions: $C_{1} \cup C_{2}, C_{1}, C_{2}$ with $C_{1}=\left|\chi^{n-1}-\chi^{n}\right|<$ cg_Ftol $\left(1+\chi^{n}\right)$ and $C_{2}=\left\|x_{n-1}-x_{n}\right\|<\operatorname{cg}$ _Ftol $\left(1+\left\|x_{n}\right\|\right)$, where $\chi^{n}$ is the cost function evaluated at the $n^{\text {th }}$-step and $x_{n}$ the corresponding argument. Increasing the value of the stop parameter generally loosens up the exit conditions of the minimization.

\subsection{Diagonalization}

The Hamiltonian diagonalization and the construction of the low-lying spectrum are performed in the module DIAG. For a given set of conserved QNs, the sector Hamiltonian $H_{\mathcal{S}}$ is diagonalized using either P-Arpack algorithm ${ }^{44,45}$ or simple Lanczos ${ }^{39}$ procedure with no re-orthogonalization, as controlled by the input variable lanc_method=arpack, lanczos. Sectors with dimensions smaller than the input value lanc_dim_threshold are diagonalized using Lapack method. The diagonalization requires to visit all the Hamiltonian sectors. If no long-range order is considered, only half of the sectors can be considered exploiting a symmetry relation between sectors with exchanged QNs, i.e. the eigenvalues of $\mathcal{S}\left[\vec{N}_{\uparrow}, \vec{N}_{\downarrow}\right]$ are identical to those of $\mathcal{S}\left[\vec{N}_{\downarrow}, \vec{N}_{\uparrow}\right]$, while the corresponding eigenvectors are identical only up to a trivial reordering. The logical variable ed_twin=True, False controls this feature. In order to further restrict the list of the analysed sectors we allow the user to indicate explicitly the sectors to solve. This operative mode is controlled by the variable ed_sectors=True,False. The QNs corresponding to the sector to analyse are read from the file Sectorsfile. Such list can also be partially enlarged by indicating the number of "neighboring" sectors to be solved, corresponding to QNs shifted by an amount ed_sectors_shift with respect to those on the user list. 


\subsection{Hamiltonian Construction and Matrix-Vector Product}

The Hamiltonian matrix construction and the related MVPs operations are contained in the module HAMILTONIAN. Different type of MVP are envisaged depending on the input parameters ed_Sparse_H and ed_total_ud which, respectively, control the storage of the Hamiltonian and the type of QNs. In order to comply with the different operational modes the MVP is abstracted into a procedure contained in the global module VARS_GLOBAL:

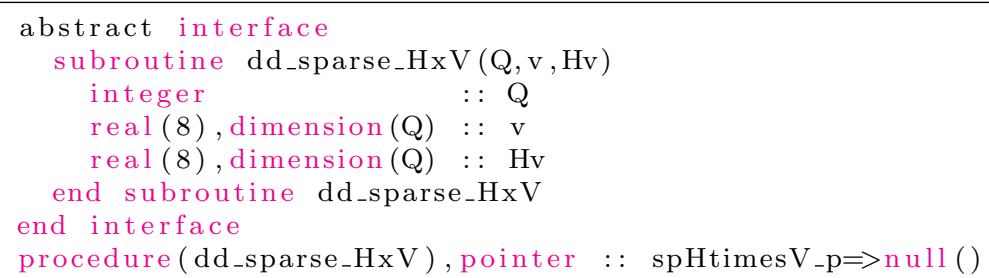

The MPI setup, the Hamiltonian construction and the association of the pointer instance spHtimesV_p to the correct MVP procedure are performed in build_Hv_sector contained in HAMILTONIAN. The matrix construction is performed in the module HAMILTONIAN_SPARSE_HxV. The sector Hamiltonian is represented by a hermitian matrix of dimension $D_{\mathcal{S}}^{2}$. The overall low connectivity of the quantum impurity models reflects in a strong sparseness of the Hamiltonian, which hosts at most $O\left(N_{s}\right) \ll D_{\mathcal{S}}$ elements on each row. We exploit the Hamiltonian decomposition (9) to minimize the memory footprint storing each matrix component separately. The diagonal term $H_{d}$ corresponds to a single vector of length $D_{\mathcal{S}}$. The tensor product structure of the hopping terms can be readily reconstructed by storing the terms $H_{\alpha \sigma}$ in a tuple of sparse matrices,. The total dimension of such tuple is at most of order $O\left(N_{u d} N_{s} \max \left(D_{\alpha \sigma}\right)\right)$, which is much smaller than $O\left(N_{s} D_{\mathcal{S}}\right)$. Finally, $H_{n d}$ is stored as a separate sparse matrix with a leading dimension $D_{\mathcal{S}}$. This component has a very sparse nature, containing a number $n \ll N_{s}$ of elements per row.

The key part of the EDIpack library is the parallel execution of the MVPs using either the memory stored Hamiltonian or the on-the-fly execution. All the MVP instances are contained in the two modules HAMILTONIAN_SPARSE_HxV and HAMILTONIAN_DIRECT_HxV. As discussed above the fundamental step in the execution of each MVP is the parallel transposition of the incoming vector. The corresponding algorithm implementation is presented below:

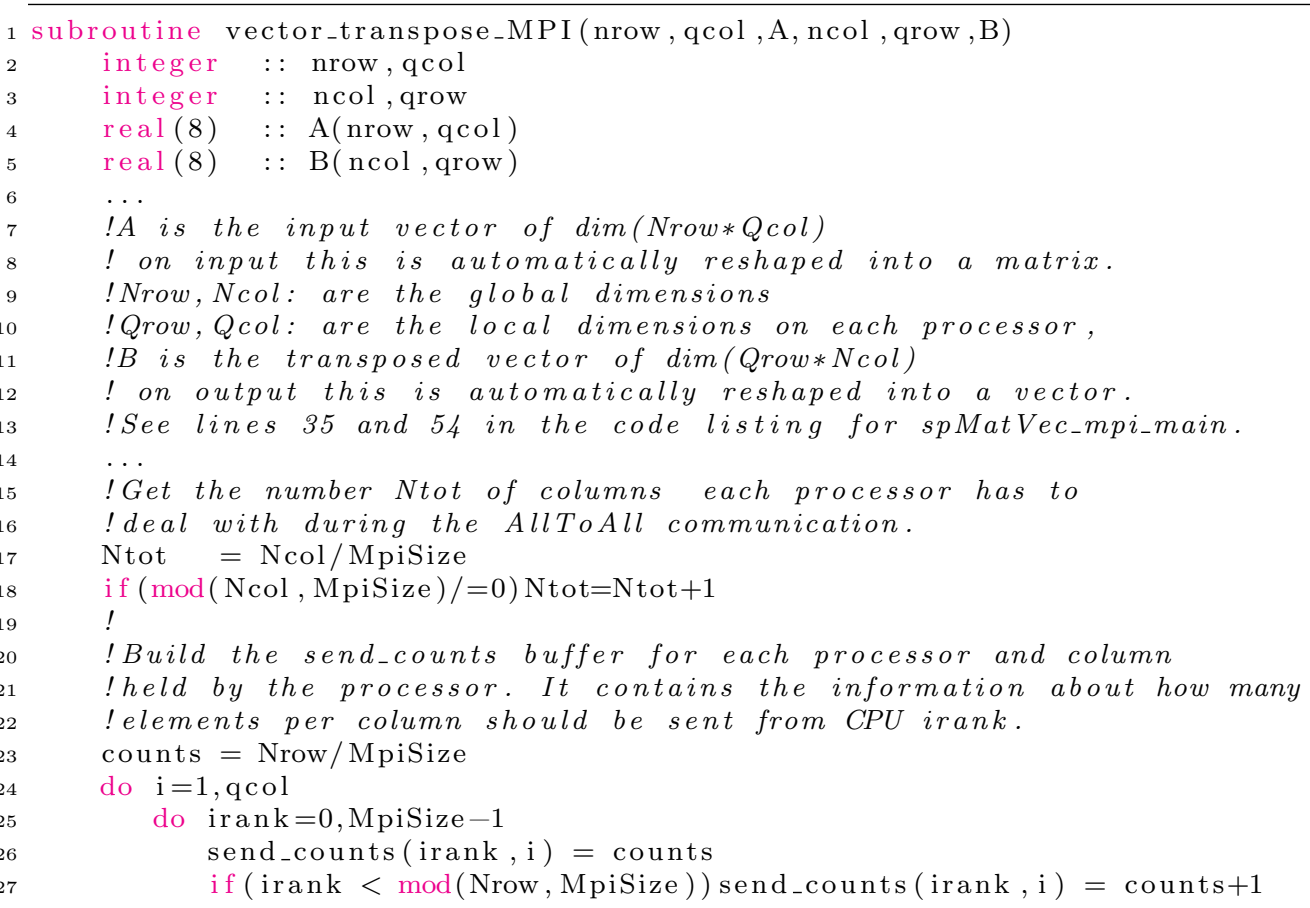




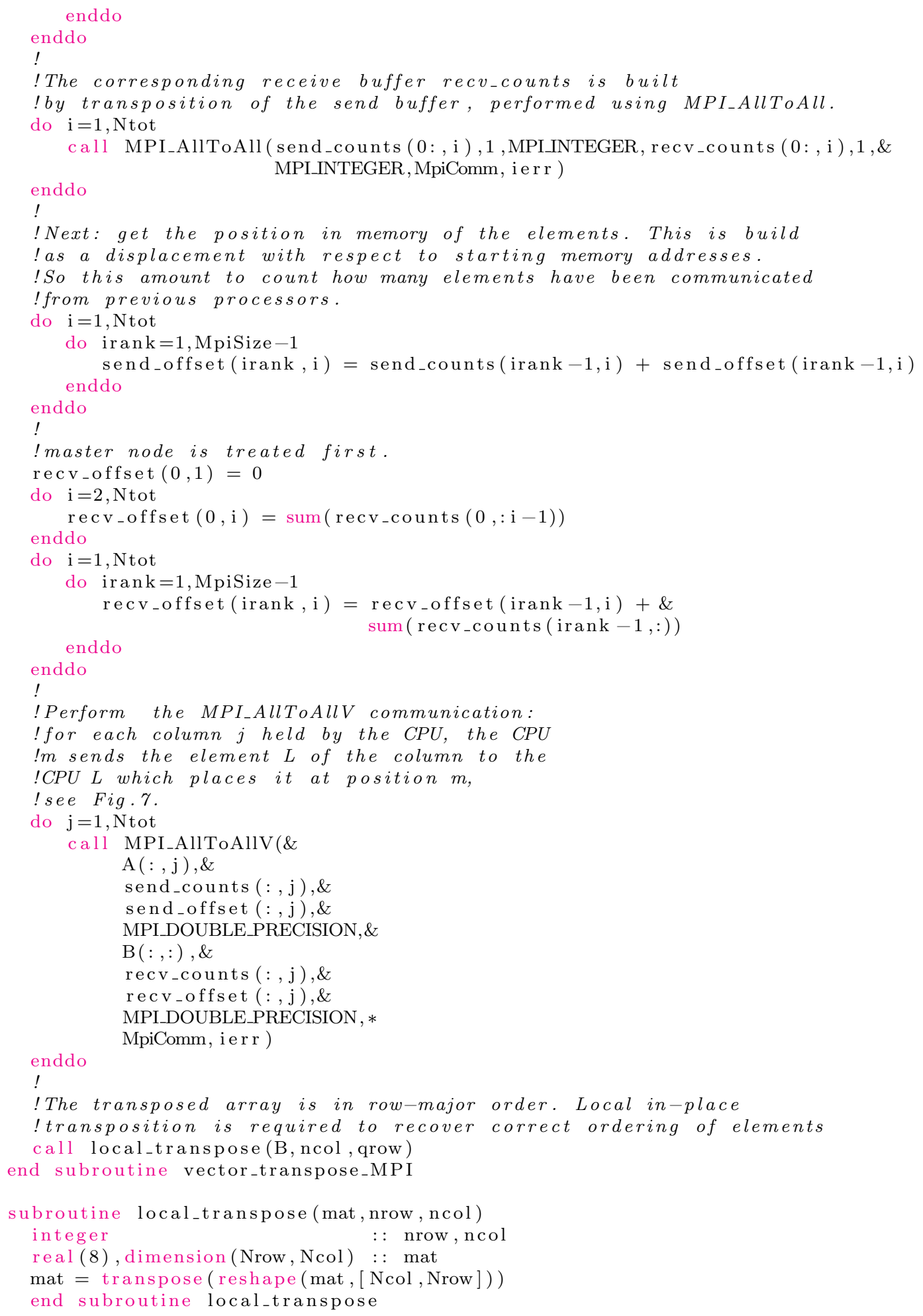

The initial part of the procedure deals with the generic determination of the input arrays required by the MPI_AllToAllV procedure. In the first section (lines 17-29) the number of elements of the source buffer to be sent by each process is determined. This information is stored in the array send_counts. By construction, 
see Fig. 7, the corresponding amount of data received in the receive buffer, i.e. recv_counts, is obtained by transposing the send_counts array itself. This is performed using MPI_AllToAll (lines 33-36). Next, the positions in memory of the send and receive elements have to be determined. These correspond to the relative displacements of the source and receive data with respect to their starting memory addresses, i.e. send_offset and recv_offset, which can be evaluated by summing over the number of elements to be sent or received by each processor, see lines $42-57$.

The matrix transposition is performed proceeding column-by-column, see lines $65-76$, repeatedly calling the MPI_AllToAllV procedure ${ }^{49}$. Note that some process may run out of columns to send because of the incommensurability of the matrix size with the number of processes (the parameter Ntot can be different from Qcol). In this case MPI2 allows to send empty arrays. The final array is however arranged in row-major mode, thus a local transposition on each process is required to reorder the data in column-major mode.

Finally, we present the implementation of the parallel MVP procedure, as described in Sec. 3.2:

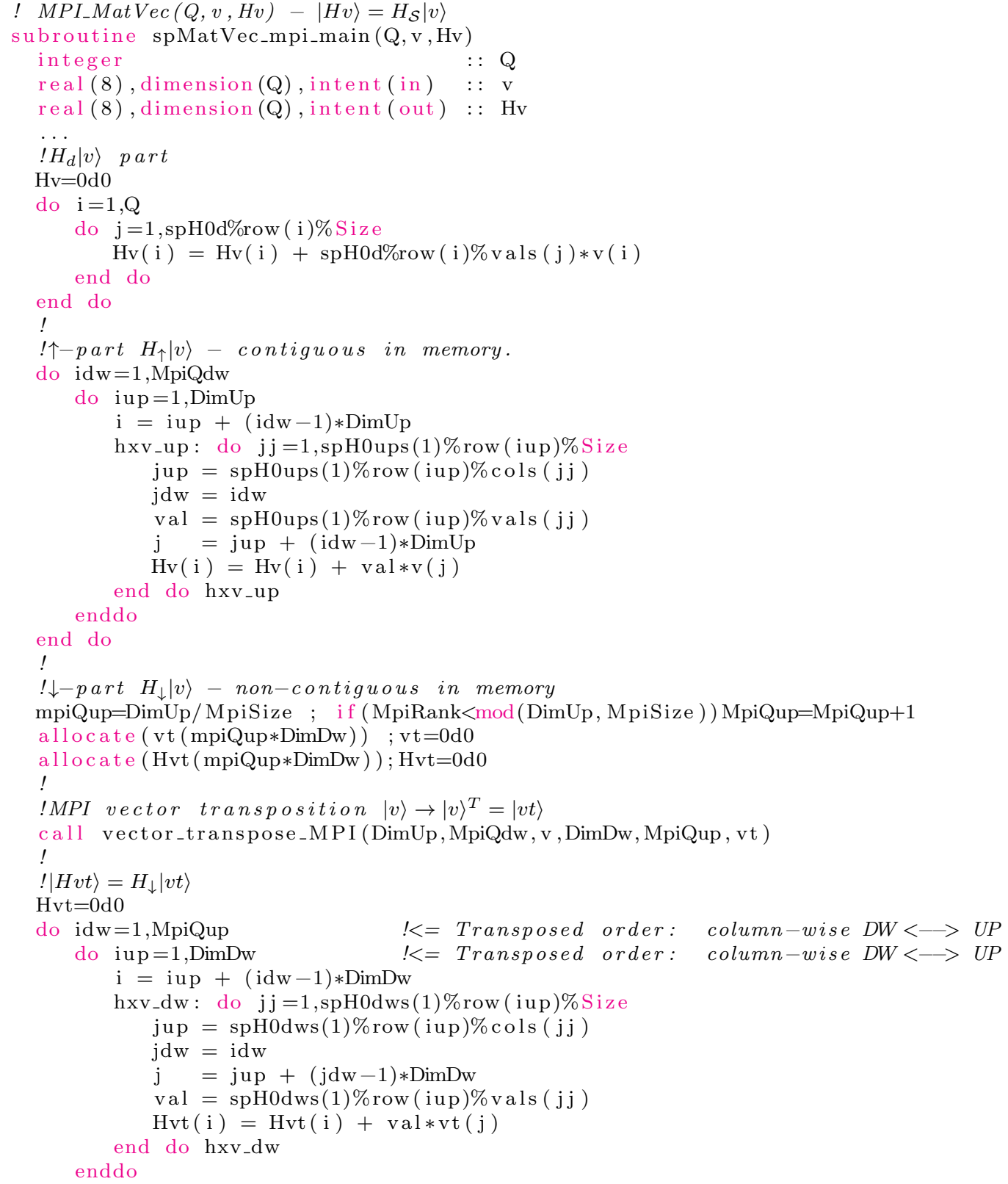




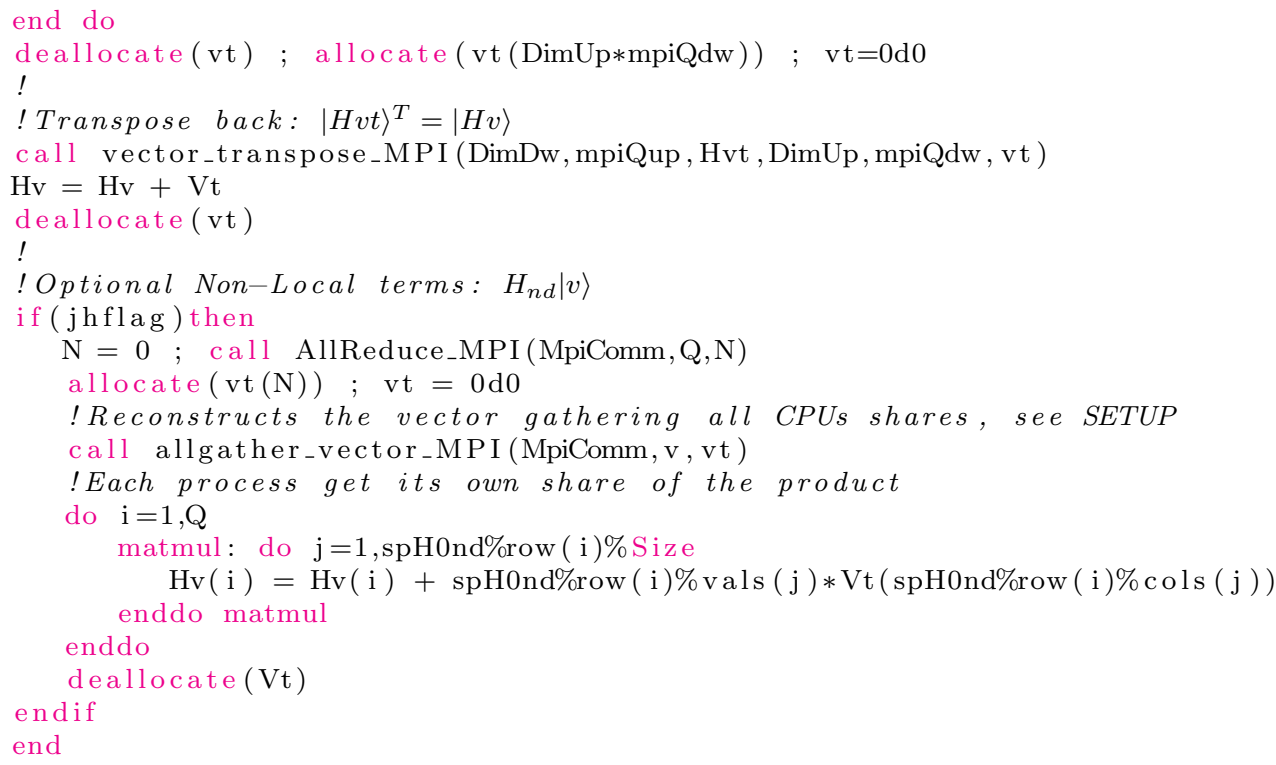

\subsection{Dynamical correlation functions}

The knowledge of the Hamiltonian spectrum, or just of its lowest part, provides the means to evaluate generic dynamical correlation functions of the form: $C_{\mathcal{O}}=\left\langle\mathcal{T}_{ \pm}\left[\mathcal{O}(t) \mathcal{O}^{+}\right]\right\rangle$, where $\mathcal{O}(t)=e^{i H t} \mathcal{O} e^{-i H t}$ is an operator in the Heisenberg representation, $\mathcal{T}_{ \pm}$is the time-ordering operator for fermions $(+)$or bosons $(-)$ and $\langle\mathcal{O}\rangle=\frac{1}{Z} \operatorname{Tr}\left[e^{-\beta H} \mathcal{O}\right], Z=\sum_{n} e^{-\bar{\beta} E_{n}}$, is the thermodynamic average. Performing a Fourier transformation and using spectral theorem, the expression for $C_{\mathcal{O}}$ can be recasted in the more convenient form:

$$
\begin{aligned}
C_{\mathcal{O}}(z) & =\left\langle\mathcal{O} \frac{1}{z-H} \mathcal{O}^{+}\right\rangle \mp\left\langle\mathcal{O}^{+} \frac{1}{z+H} \mathcal{O}\right\rangle \\
& =\frac{1}{Z} \sum_{n} e^{-\beta E_{n}}\left\langle\psi_{n}\left|\mathcal{O} \frac{1}{z-H} \mathcal{O}^{+}\right| \psi_{n}\right\rangle \mp\left\langle\psi_{n}\left|\mathcal{O}^{+} \frac{1}{z+H} \mathcal{O}\right| \psi_{n}\right\rangle \\
& =\frac{1}{Z} \sum_{n} e^{-\beta E_{n}} \sum_{m} \frac{\left\langle\psi_{n}|\mathcal{O}| \psi_{m}\right\rangle\left\langle\psi_{m}\left|\mathcal{O}^{+}\right| \psi_{n}\right\rangle}{z-\left(E_{m}-E_{n}\right)} \mp \frac{\left\langle\psi_{n}\left|\mathcal{O}^{+}\right| \psi_{m}\right\rangle\left\langle\psi_{m}|\mathcal{O}| \psi_{n}\right\rangle}{z+\left(E_{m}-E_{n}\right)}
\end{aligned}
$$

where $z \in \mathbb{C},\left|\psi_{n}\right\rangle$ are the eigenstates of the Hamiltonian $H$ and $E_{0}$ is the groundstate energy. A key simplification in the evaluation of such dynamical correlation function is obtained using the Lanczos procedure. Introducing a suitable Krylov basis the Hamiltonian matrix takes a tri-diagonal form, which allows to evaluate an arbitrary number of terms in the sum over the internal excited states $\left|\psi_{m}\right\rangle$. Let's con-

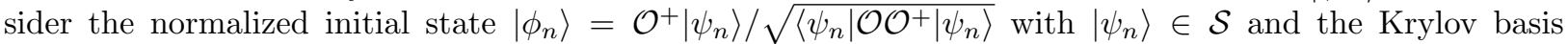
$\mathcal{K}_{N}\left(\left|\phi_{n}\right\rangle\right)=\left\{\left|\phi_{n}\right\rangle, H\left|\phi_{n}\right\rangle, \ldots, H^{N}\left|\phi_{n}\right\rangle\right\} \equiv\left\{\left|v_{0}^{n}\right\rangle,\left|v_{1}^{n}\right\rangle, \ldots,\left|v_{N}^{n}\right\rangle\right\}$ with $1 \ll N \ll \mathcal{D}_{\mathcal{S}}$. Any eigenstate $\left|\psi_{n}\right\rangle$ is decomposed along the $\mathcal{K}_{N}\left(\left|\phi_{n}\right\rangle\right)$ basis as: $\left|\psi_{n}\right\rangle=\sum_{i}\left\langle v_{i}^{n} \mid \psi_{n}\right\rangle\left|v_{i}^{n}\right\rangle=\sum_{i} a_{i}^{n}\left|v_{i}^{n}\right\rangle$. Using this decomposition, we can evaluate the operator components $\left\langle\psi_{m}\left|\mathcal{O}^{+}\right| \psi_{n}\right\rangle=\sqrt{\left\langle\psi_{n}\left|\mathcal{O O}^{+}\right| \psi_{n}\right\rangle} a_{m}^{n}$ and thus:

$$
\left\langle\psi_{n}\left|\mathcal{O} \frac{1}{z-H} \mathcal{O}^{+}\right| \psi_{n}\right\rangle \simeq\left\langle\psi_{n}\left|\mathcal{O O}^{+}\right| \psi_{n}\right\rangle \sum_{m=1}^{N} \frac{\left|a_{m}^{n}\right|^{2}}{z-\left(E_{m}-E_{n}\right)}
$$

Repeating the same decomposition for each term in Eq. 18, we obtain the following approximation to 
$G_{\mathcal{O}}:$

$$
\begin{aligned}
& C_{\mathcal{O}}(z)=\frac{1}{Z} \sum_{n} e^{-\beta E_{n}} C_{\mathcal{O}}^{n}(z) \\
& C_{\mathcal{O}}^{n}(z) \simeq \sum_{m=1}^{N} \frac{\left\langle\psi_{n}\left|\mathcal{O} \mathcal{O}^{+}\right| \psi_{n}\right\rangle\left|a_{m}^{n}\right|^{2}}{z-\left(E_{m}-E_{n}\right)} \mp \frac{\left\langle\psi_{n}\left|\mathcal{O}^{+} \mathcal{O}\right| \psi_{n}\right\rangle\left|a_{m}^{n}\right|^{2}}{z+\left(E_{m}-E_{n}\right)}
\end{aligned}
$$

In many concrete situations one has to deal with non-diagonal dynamical correlation functions, e.g.: $C_{\mathcal{A B}}(z)=\left\langle A^{+} \frac{1}{z-\left(H-E_{0}\right)} B\right\rangle$, which can not be treated by the procedure outlined above. A simple solution is to consider auxiliary operators of the form $\mathcal{O}=\mathcal{A}+\mathcal{B}$ and $\mathcal{P}=\mathcal{A}-i \mathcal{B}$. Using simple algebra it is then straightforward to obtain the desired function $C_{\mathcal{A B}}$ from the evaluation of $C_{\mathcal{O}}$ and $C_{\mathcal{P}}$ :

$$
C_{\mathcal{A B}}=\frac{1}{2}\left[C_{\mathcal{O}}+C_{\mathcal{P}}-(1+i) C_{\mathcal{A}}-(1+i) C_{\mathcal{B}}\right]
$$

The evaluation of the impurity Green's functions $G_{\alpha \beta}=\left\langle\mathcal{T}_{ \pm}\left[c(t) c^{+}\right]\right\rangle$is executed in GREENS_NORMAL. Similarly, spin, charge, excitonic and pair susceptibilities functions are evaluated in the modules CHI_SPIN, CHI_DENS, CHI EXCT and CHI_PAIR. All the dynamical correlation functions are evaluated along the imaginary and real-frequency axis. The susceptibilities are also evaluated in imaginary time. The most time consuming part of this step is the construction of the Krylov basis $\mathcal{K}_{N}\left(\mathcal{O}\left|\psi_{n}\right\rangle\right)$ associated to each of the states $\left|\psi_{n}\right\rangle$ of the spectrum determined in the diagonalization step. This ultimately requires to perform a number $N$ of MVP for any target state $\left|\psi_{n}\right\rangle$. This task is handled using parallel algorithm discussed above, greatly reducing the computational cost of this operation. The order of the Krylov basis is controlled by the input variable lanc_gfniter.

The evaluation of a dynamical correlation function introduces an intrinsic small memory bottleneck, related to the non-local nature of the operator application $\left|\phi_{n}\right\rangle=\mathcal{O}\left|\psi_{n}\right\rangle$, which in our implementation is performed by the master node and subsequently distributed to the other processes.

\subsection{Observables}

Local observables, such as occupation, double occupancies and local energy, are evaluated in the module OBSERVABLES using thermal average $\langle\mathcal{O}\rangle=\operatorname{Tr} e^{-\beta H} \mathcal{O} / Z$. Having access to the lowest part of the spectrum we can evaluate the first terms in the thermodynamic trace: $\langle\mathcal{O}\rangle=\frac{1}{Z} \sum_{n} e^{-\beta E_{n}}\left\langle\psi_{n}|\mathcal{O}| \psi_{n}\right\rangle$, where $\left|\psi_{n}\right\rangle$ are the eigenstates of the system.

\subsection{Main}

The principal interfaces to the EDIpack library are contained in the MAIN module, which contains two procedures. The ?init_solver aims to initialize the ED solver, by performing a series of compatibility checks, allocating the required static memory and initializing the bath class either performing a generic guess or reading the bath from a file (specified by the input parameter Hfile). This procedure accepts as only input the user defined bath, i.e. an array of real numbers whose dimension should conform the output of ?get_bath_dimension, see Table 2 .

The ED solver is interfaced by the procedure ?solve, which requires as input the user bath array and the local non-interacting Hamiltonian term $H^{l o c}$ array. The local Hamiltonian is used to setup of the structure of the impurity problem. A call to ?solve performs all the calculations necessary to solve the quantum impurity problem for the input bath. All the dynamical correlations and observables are stored in the memory and accessible via suitable input/output procedures, see Table 2.

In presence of inequivalent atoms or sites the problem is solved by independently diagonalizing the impurity problem of each atom or site. The user should allocate a rank-2 array to store the baths where the first dimension corresponds to the number $N_{\text {ineq }}$ of inequivalent atoms or sites. In analogy, the local noninteracting Hamiltonian should correspond to a rank- 5 array in which the first dimension should be $N_{\text {ineq }}$. Upon call to ?solve, each of the $N_{\text {ineq }}$ quantum impurity problems is solved. Using the optional input parameter mpi_lanc the user can select parallel execution either on the inequivalent sites (mpi_lanc=False and default) or on the diagonalization algorithm (mpi_lanc=True). 


\section{Installation and usage}

The EDIpack library is released under open source GNU GPL license. The source code is publicly available at the GitHub on-line repository: github.com/QcmPlab/EDIpack. For the installation few dependencies have to be satisfied. In particular the code relies on the open source scientific library SciFortran, available at github.com/QcmPlab/SciFortran:

- Fortran compiler with support to major fortran 90/2003 standard, e.g. GNU gfortran version > 9.0.0 or Intel ifort > 13.0.0.

- CMake $>=3.0 .0$

- MPI

- SciFortran

Additional dependencies for the Python binding are:

- Python, version $>=3.6 .0$

- Pkgconfig

- Numpy

- Mpi4py

\subsection{Installation}

The source code can be downloaded or cloned from the official GitHub website. The build system is based on cmake. The library can be loaded into the operative system either via the provided shell script or as an environment module. Should all the dependencies be satisfied and assuming to be in the main library directory, the compilation procedure is:

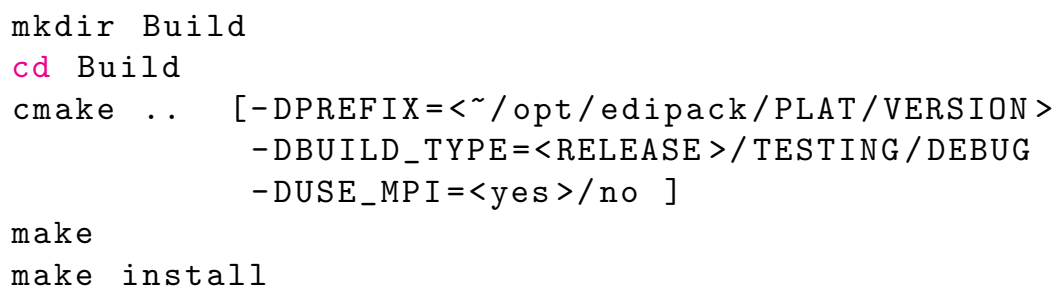

The Python binding can be installed once the library has been loaded in the system using either:

export $F 90=m p i f 90$

pip install.

We tested installation of the EDIpack library in different platforms, e.g. common Linux distribution, Unix/OSX systems and dedicated HPC.

\subsection{Basic usage}

The goal of EDIpack is to provide an efficient and scalable method to solve quantum impurity problems. As such, use of the library requires the user to write a suitable driver program. Two commented examples, using the Fortran and Python interfaces respectively, are included in the test directory of the library. These test programs aim to solve the DMFT equations for the single band Hubbard model on the Bethe lattice. The provided codes can be used as templates for other specific cases (see github.com/QcmPlab/DriverDatabase). 


\subsubsection{Fortran API}

The EDIpack library is entirely written in Fortran. The internal structure of the library is opaque to the user, so that only a number of variables and procedures are available while the remaining parts are not directly accessible by the user. The interface is implemented by the outmost module EDIPACK, which gives access to all the necessary procedures and control variables, see Table 1 (first column) and Table 2. The Fortran binding can be loaded in a program using the statement:

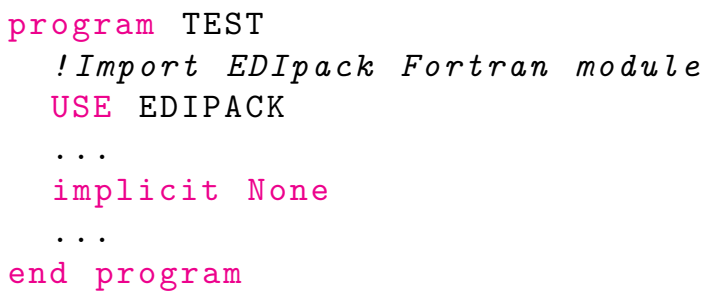

Because Fortran does not allow namespace resolution, all the Fortran procedures are preceded by the string ed, see Table 2 .

\subsubsection{Python API}

Use of the EDIpack library within Python is possible through suitable API. The Python binding is built using a specific interface module edi2py generated by numpy.f2py from a suitable Fortran layer, i.e. a collection of Fortran interfaces linking to the Fortran API. Finally, the Python module edipy is generated operating on the edi2py interface and exploiting "duck typing" to ensure full compatibility between Fortran and Python API. The Python binding can be loaded as:

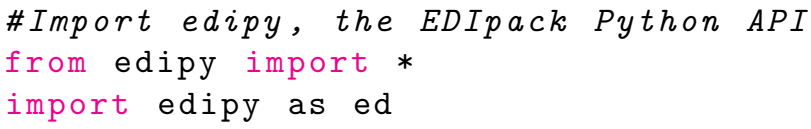

The Python procedures are preceded by the namespace of the module as edipy. and can be conveniently renamed as in this example or in the available test code.

\subsubsection{MPI Parallel interface}

The EDIpack library relies on distributed memory MPI implementation ${ }^{49}$. The MPI specific code is included in the library by means of pre-processing instructions identified by the macro _MPI. MPI support is activated by default in the installation. The library supports either parallel (MPI initialized) or serial (MPI not initialized) programs, see Sec. 7.2.

In order to keep the interface as simple as possible, the MPI support is completely implicit. The parallel execution of any EDIpack procedure is activated once the MPI environment has been initialized in the calling program. To this end, a system query MPI_Initialized is used to determine whether or not the user activated MPI. If true, MPI instructions are executed and internal MPI setup is performed by calling ed_set_MpiComm, contained in the module VARS_GLOBAL. Thus, the user is only required to initialize (MPI_Init) and finalize (MPI_Finalize) the MPI environment in the calling program in order to perform parallel calculations. Note that in the Python module mpi4py these steps are automatic.

\subsubsection{Workflow}

Although the calling program can differ substantially case by case, a general workflow for the use of EDIpack can be identified. Here we briefly discuss such workflow for the case of single-impurity problem. The case of inequivalent atoms or sites requires to change the dimension of allocated array as discussed in Table 2.

The first part of the program requires to load EDIpack and, if required, MPI should be initialized. Next, the input file has to be read with a call to the procedure ?read_input. The only input argument of this procedure is the name of the input file. Should the file not exist in the execution directory, a suitable input file with prefix used. is created, using default values for all the input parameters along with a short 
comment. After renaming, this file can be used as a starting point to setup the calculation. The EDIpack control variables can additionally be controlled from command line with the simple syntax Variable=Value appended after the program call. Moreover, a used version of the input file with prefix used., containing the variables actually used during the run, is written after each call to the main solver. An overview of the input variables of the EDIpack library is reported in Table 1. The introduction of many input parameters enables a higher level of control over the library. Yet, their number can be frightening for the inexperienced user. To this end, all the variables are initialized to a default value which is suitable to perform calculation at zero temperature for a single band model. In the first part of Table 1 we report the most relevant input variables, controlling important aspects of the impurity problem including external parameters such as temperature, interaction strength, chemical potential, etc. In the second part table we list the remaining and more specific input variables, which can be used to reach a finer tuning of the library performances or to adapt the functioning to specific cases.

After this step the user is required to setup some properties specific to the problem. For the DMFT application this usually reduces to generating the necessary density of states $D(e)$ or the tight-binding Hamiltonian $H(k)$. The local part of the non-interacting Hamiltonian $H^{l o c}$ should be allocated and defined. The EDIpack library requires this input to be a rank-4 dble array of shape [Nspin, Nspin, Norb, Norb] . In addition, local dynamical function in the Matsubara axis, i.e. the local Green's function $G\left(i \omega_{n}\right)$, or the impurity self-energy $\Sigma\left(i \omega_{n}\right)$, should be allocated with the correct dimensions. Note that EDIpack requires these arrays to have shape [Nspin,Nspin,Norb,Norb,Lmats], where Nspin, Norb and Lmats are input variables. Real-axis analogs of such functions can also be allocated and used, although these do not necessarily participate to the self-consistency procedure.

Terminated this part the user should initialize the solver. This task requires two steps. The first is to query the EDIpack library to know the dimension of the rank-1 dble array storing the bath parameters and used in the reverse communication implementation. This information is retrieved with a call to ?get_bath_dimension, which returns such dimension as an integer. The second step, is to call ?init_solver to initialize the ED solver with the user bath array as the only input. On return, the input array contains the bath parameters. These are either read from the file Hfile.restart, if it exists in the executing directory, or guessed from a default flat density of states controlled by the input variables ed_hw_bath and ed_offset_bath, see Table 1.

At this stage the user is ready to call the ED solver ?solve whose only inputs are the bath and the local Hamiltonian arrays. Within the DMFT framework this step is usually performed inside a self-consistency cycle, which in its simplest form just corresponds to an iterative one. At each loop the user calls the EDIpack ED solver, which solves the effective quantum impurity problem determined by the user provided bath. This is the most time-consuming part of the cycle. The results of the calculations are stored in the memory up to a subsequent call of the solver. Local dynamical impurity functions and many observables can be retrieved from the memory using suitable procedures, see Table 2. In particular the impurity matsubara self-energy, used within the self-consistency, is retrieved with a call to ?get_sigma_matsubara.

Next, the user is required to perform the self-consistency condition, which is highly dependent on the specific problem. This part of the calling program is completely free and independent of EDIpack.

Finally, and in order to close the DMFT loop the user should update the bath according to the outcome of the self-consistency equation. This step can be performed using the conjugate gradient minimization provided with EDIpack i.e. calling ?chi2_fitgf. Yet, other methods can in principle be adopted. The updated bath parameters will be used as input of the ED solver, until convergence is achieved.

\subsubsection{Output}

All the output of the EDIpack library is written in plain text and distributed among different files with the extension .ed. The output can be divided in three main groups.

The first group contains all the dynamical impurity functions, including the Green's functions, the selfenergies and the susceptibilities. The input variables ed_print_Sigma, ed_print_G, ed_print_GO can be used to allow or suppress write out of, respectively, the self-energy, the impurity and the non-interacting Green's functions. The quantities in this group are written to files of the form impFUnC_l $\alpha \beta \_s \sigma_{-} \mathrm{x}$. ed where FUNC=GO, G, Sigma indicates the non-interacting Green's function, the impurity one and the self-energy, 
$\alpha, \beta$ are orbital indices, $\sigma=1,2$ corresponds to the spin index and $\mathrm{x}=\mathrm{iw}$, realw, respectively for the Matsubara and real-axis frequency. Each file contains three columns, in order: the frequency, the imaginary part and the real part.

The second group of outputs contains the values of several static quantities. In order to ease the data analysis with non-blocking shell commands such quantities are stored in multi-column files. In order to avoid problems with data processing the columns headers are reported in separate files using the same name of the data file but with the suffix _info.ed. The most important observables and other significant quantities are contained in the files observables_all.ed (which subsequently stores variables from all calls to the solver) and observables_last.ed (which contains the values from the last call). The contributions to the local energy $\langle H\rangle$ are written in columns in the file energy_last.ed. The excitonic order parameters $P^{m}=\left\langle c_{\alpha r}^{+} \sigma_{r s}^{m} c_{\beta s}\right\rangle$, with $\sigma^{m=0, z}$ Pauli matrices, are written to the file exciton_last.ed.

The third group contains all the remaining files. These include the bath used in the actual calculation, written to Hfile.used, the restart files Hfile.restart and SectorFile.restart, respectively containing the bath resulting from the fit and the list of sectors QNs contributing to the evaluated spectrum. The .restart files can be used to restart a given calculation. The list of the eigenvalues determined during the diagonalization procedure is written to eigenvalues_list.ed. Similarly, a summary of the states list is written to the file state_list.ed. Some of the parameters used in the calculations, conventionally used for data analysis, are reported column-wise in the file parameters_last.ed. The result of the fit is written to files of the form chi2fit_results_orb $\alpha_{-} \mathbf{s} \sigma$.ed, with $\alpha$ orbital index and $\sigma=1,2$ the spin index. Similarly, the input function $X\left(i \omega_{n}\right)$ and the fitted function $X^{A n d}\left(i \omega_{n} ;\{V, h\}\right)$ resulting from the fit are written to files of the form fit_X_orb $\alpha_{-} \mathbf{s} \sigma$.ed, with $\alpha$ and $\sigma$ as above and $\mathrm{X}=\mathrm{Delta}$, Weiss according to the value of cg_scheme.

Finally, the EDIpackand the SciFortran GIT SHA1 identifiers used in the current calculation are reported, respectively, in the files EDIPACK_version.inc and scifor_version.inc.

\section{Acknowledgements}

We acknowledge fruitful discussions with G. Sangiovanni, A. Sartori, H. Choi, A. Valli, S. Adler and M. Chatzieleftheriou. A.A. and M.C. acknowledge support from H2020 Framework Programme, under ERC Advanced Grant No. 692670 FIRSTORM and financial support from MIUR PRIN 2015 (Prot. 2015C5SEJJ001) and SISSA/CNR project "Superconductivity, Ferroelectricity and Magnetism in bad metals" (Prot. 232/2015). L.C. acknowledges financial support by the Deutsche Forschungsgemeinschaft (DFG, German Research Foundation) under Germany's Excellence Strategy through Würzburg-Dresden Cluster of Excellence on Complexity and Topology in Quantum Matter - ct.qmat (EXC 2147, project-id 390858490). G.M. acknowledges support of the FNS/SNF through an Ambizione grant and partly supported from the European Research Council (ERC-319286-QMAC). L.d.M is supported by the European Commission through the ERC-CoG2016, StrongCoPhy4Energy, GA No 724177.

\section{Appendix A: Input Variables}

Here is reported a list of the global input variables of the library. The input parameters are arranged into the following table, as discussed in Sec. 7.2.4. Note that only a subset of the input parameters are transparent to the user, i.e. can be directly accessed from the calling program. These variables are indicated with a symbol in the first column of the table. In the columns from $2^{\text {nd }}$ to $4^{\text {th }}$ we indicate, respectively, the variable name, the type and the default value. Finally, In the last column we provide a short description of the variable purpose. 
Table 1: List of the EDIpack input variables.

\begin{tabular}{|c|c|c|c|c|}
\hline & Variable & Type & Default & Description \\
\hline & & & \multicolumn{2}{|c|}{ Most relevant control variables } \\
\hline$\circ$ & Norb & Int & 1 & Number of impurity orbitals. \\
\hline \multirow[t]{2}{*}{$\circ$} & Nspin & Int & 1 & $\begin{array}{l}\text { Number of spin channels treated independently. Nspin=1 enforces } \\
\text { non-magnetic solution. }\end{array}$ \\
\hline & Nbath & Int & 6 & $\begin{array}{l}\text { Number of bath sites. See bath_type for information about resulting } \\
\text { total number of electronic levels } N_{s} \text {. }\end{array}$ \\
\hline$\circ$ & bath_type & Char & "normal" & $\begin{array}{l}\text { Set the bath type: normal: } N_{s}=\operatorname{Norb}(1+\text { Nbath }) \text {, hybrid: } \\
N_{s}=\text { Norb + Nbath, and replica: } N_{s}=\operatorname{Norb}(1+\text { Nbath }) \text {, See Sec. } 6.4 \text {. }\end{array}$ \\
\hline$\circ$ & Uloc & Dble & 2.0 & $\begin{array}{l}\text { Local intra-orbital interactions } U \text {, different values per orbitals can be } \\
\text { defined. }\end{array}$ \\
\hline$\circ$ & Ust & Dble & 0.0 & $\begin{array}{l}\text { Local inter-orbital interaction } U^{\prime} \text {, with opposite spin orientations. } \\
\text { For the Kanamori interaction this must be set to Uloc }-2 \mathrm{Jh} \text {, with Jh } \\
\text { the Hund's coupling. The value } U^{\prime \prime} \text { for equal spin orientation is } \\
\text { automatically set by the difference Ust - Jh. }\end{array}$ \\
\hline$\circ$ & $\mathrm{Jh}$ & Dble & 0.0 & Hund's coupling. \\
\hline$\circ$ & $\mathrm{Jx}$ & Dble & 0.0 & Spin-Exchange coupling constant. \\
\hline$\circ$ & $\mathrm{Jp}$ & Dble & 0.0 & Pair-Hopping coupling constant. \\
\hline$\circ$ & beta & $\mathrm{Dble}$ & 1000 & $\begin{array}{l}\text { Inverse temperature. For } T=0 \text { calculations this value sets a } \\
\text { discretization of the Matsubara frequencies. }\end{array}$ \\
\hline \multirow[t]{2}{*}{$\circ$} & $\mathrm{xmu}$ & Dble & 0.0 & $\begin{array}{l}\text { Chemical potential. This parameter sets the average occupations of } \\
\text { the impurity. If HFMODE=True this value contains the Hartree shift, i.e. } \\
\text { xmu }=0.0 \text { sets the half-filling for a particle-hole symmetric case. }\end{array}$ \\
\hline & HFmode & Bool & true. & $\begin{array}{l}\text { If True the Hartree shift is included in the interaction, e.g. } \\
U\left(n_{\uparrow}-1 / 2\right)\left(n_{\downarrow}-1 / 2\right) \text {. In this case zero chemical potential } \\
\text { corresponds to half-filling for a particle-hole symmetric system. }\end{array}$ \\
\hline$\circ$ & Lmats & Int & 4096 & Number of Matsubara frequencies. \\
\hline$\circ$ & Lreal & Int & 5000 & Number of real-axis frequencies \\
\hline$\circ$ & eps & Dble & 0.01 & Real-axis broadening. \\
\hline$\circ$ & wini, wfin & Dble & $-5,5$ & Real-axis frequency range. \\
\hline$\circ$ & Nloop & Int & 100 & Maximum number of allowed iterations in the DMFT solution. \\
\hline$\circ$ & dmft_error & $\mathrm{Dble}$ & $1 e-5$ & Convergence threshold for the DMFT iterative cycle. \\
\hline$\circ$ & Nph & Int & 0 & Maximum number of phonons. \\
\hline$\circ$ & g_ph & Dble & 0.0 & Electron-Phonon coupling. \\
\hline$\circ$ & w0_ph & Dble & 0.0 & Holstein phonon frequency. \\
\hline \multirow[t]{5}{*}{$\circ$} & xmin, $x \max$ & Dble & $-3,3$ & Phonon probability distribution spatial range. \\
\hline & chispin_flag & Bool & false. & Flag to include the evaluation of Spin-Spin susceptibilities. \\
\hline & chidens_flag & Bool & false. & Flag to include the evaluation of Density-Density susceptibilities. \\
\hline & chipair_flag & Bool & false. & Flag to include the evaluation of $s$-wave Pair susceptibilities. \\
\hline & chiexct_flag & Bool & false. & Flag to include the evaluation of Excitonic susceptibilities. \\
\hline
\end{tabular}


Table 1: List of the EDIpack input variables.

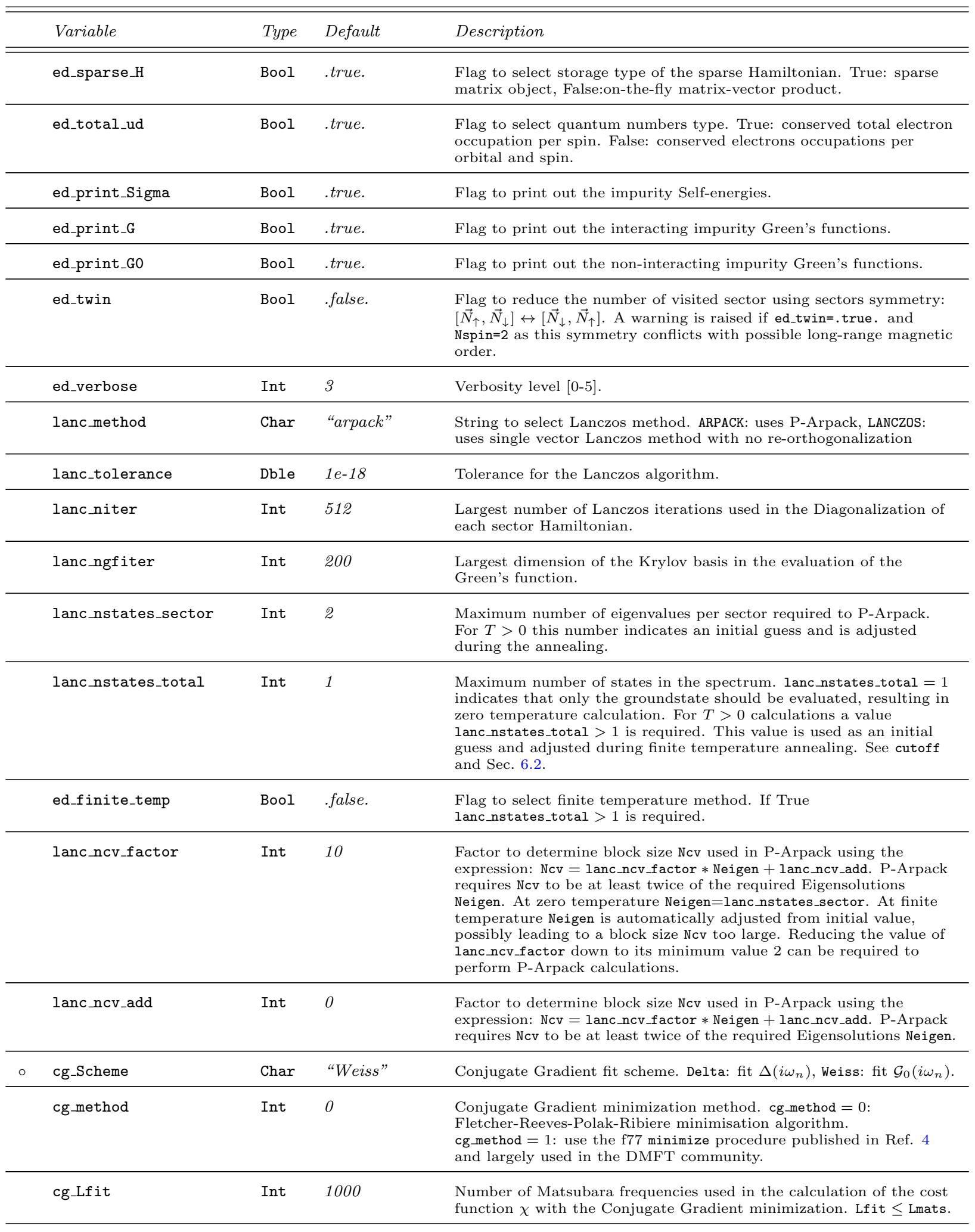


Table 1: List of the EDIpack input variables.

\begin{tabular}{|c|c|c|c|c|}
\hline & Variable & Type & Default & Description \\
\hline & cg_grad & Int & 0 & $\begin{array}{l}\text { Type of gradient evaluation. cg_grad }=0: \text { Analytic, cg_grad }=1: \\
\text { Numeric. If cg_method }=1 \text { then use of cg_grad }=1 \text { is enforced as this } \\
\text { method does not support analytic gradient calculation. }\end{array}$ \\
\hline \multicolumn{5}{|c|}{ More specific control variables, less frequently changed } \\
\hline$\circ$ & sb_field & Dble & 0.0 & Small symmetry-breaking field. \\
\hline \multirow[t]{7}{*}{$\circ$} & Nsuccess & Int & 1 & $\begin{array}{l}\text { Minimum number of repeated iterations below threshold required to } \\
\text { reach convergence. }\end{array}$ \\
\hline & cutoff & $\mathrm{Dble}$ & $1 e-9$ & $\begin{array}{l}\text { Cutoff for the Boltzmann factor in the spectral summation, i.e. } \\
e^{-\beta\left(E-E_{0}\right)}<\text { cutoff. See Sec. } 6.2 \text {. }\end{array}$ \\
\hline & gs_threshold & $\mathrm{Dble}$ & $1 e-9$ & $\begin{array}{l}\text { Energy threshold for the groundstate degeneracy. Eigenstates of } \\
\text { energy } E \text { within }\left|E-E_{0}\right|<\text { gs_threshold are considered as } \\
\text { degenerate groundstates. }\end{array}$ \\
\hline & ed_sectors & Bool & false. & $\begin{array}{l}\text { This flag is used to reduce the number of sectors investigated during } \\
\text { the construction of the spectrum. Only the sectors with QNs listed in } \\
\text { the file SectorFile and those with plus and minus ed_sectors_shift } \\
\text { are considered. }\end{array}$ \\
\hline & ed_sectors_shift & Int & 1 & $\begin{array}{l}\text { A shift of the QNs for the list of sectors read from SectorFile is } \\
\text { ed_sectors=.true.. The list is enlarged including the QNs obtained } \\
\text { by applying a shift } \pm 1, \ldots, \pm \text { ed_sectors_shift to each element of the } \\
\text { list. }\end{array}$ \\
\hline & ed_hw_bath & $\mathrm{Dble}$ & 2.0 & $\begin{array}{l}\text { The half-bandwidth for the bath initialization using discretized flat } \\
\text { density of states. }\end{array}$ \\
\hline & ed_offset_bath & $\mathrm{Dble}$ & 0.1 & $\begin{array}{l}\text { Energy offset for the initialization of the diagonal terms in replica } \\
\text { bath. A small value randomly chosen in the interval } \\
\epsilon \in[\text {-ed_offset_bath : ed_offset_bath] is added to the diagonal terms } \\
\text { of the bath to avoid possible spectral degeneracies. }\end{array}$ \\
\hline ० & Lpos & Int & 100 & $\begin{array}{l}\text { Number of points used to obtain the phonon probability distribution } \\
\text { function. }\end{array}$ \\
\hline$\circ$ & nread & $\mathrm{Dble}$ & 0.0 & $\begin{array}{l}\text { Target density for fixed density calculations. Use the procedure } \\
\text { ed_search_variable to adjust chemical potential xmu so that local } \\
\text { occupation is } \mid n-\text { nread } \mid<\text { nerr. }\end{array}$ \\
\hline & nerr & $\mathrm{Dble}$ & $1 e-4$ & Error threshold for fixed-density calculations. \\
\hline & ndelta & $\mathrm{Dble}$ & 0.1 & $\begin{array}{l}\text { Initial step for the variation of the chemical potential in fixed-density } \\
\text { calculations. }\end{array}$ \\
\hline & ncoeff & $\mathrm{Dble}$ & $1 d 0$ & $\begin{array}{l}\text { A multiplier for the initial ndelta as read from a file: } \\
\text { ndelta } \rightarrow \text { ndelta } * \text { ncoeff. }\end{array}$ \\
\hline & ed_solve_offdiag_gf & Bool & false. & $\begin{array}{l}\text { Force the calculation of the off-diagonal Green's functions. True if } \\
\text { bath_type } \neq \text { normal }\end{array}$ \\
\hline & ed_all_G & Bool & false. & $\begin{array}{l}\text { Flag to evaluate all components of the impurity Green's functions, } \\
\text { irrespective of the symmetries of the problem when using } \\
\text { bath_type=replica. If False only the Green's functions corresponding } \\
\text { to non-vanishing components of the bath Hamiltonian } h_{\alpha \beta \sigma}^{\nu} \text { are } \\
\text { evaluated. }\end{array}$ \\
\hline & lanc_nstates_step & Int & 2 & $\begin{array}{l}\text { Number of states to be added to lanc_nstates_sector for each sector } \\
\text { during finite temperature annealing of the state list. See Sec. Sec. 6.2. }\end{array}$ \\
\hline & lanc_dim_threshold & Int & 1024 & $\begin{array}{l}\text { Dimension threshold below which Lapack diagonalization method is } \\
\text { used to determine eigensolutions. }\end{array}$ \\
\hline
\end{tabular}


Table 1: List of the EDIpack input variables.

\begin{tabular}{|c|c|c|c|c|}
\hline & Variable & Type & Default & Description \\
\hline & cg_Niter & Int & 500 & $\begin{array}{l}\text { Maximum number of iteration for the Conjugate Gradient } \\
\text { minimization. }\end{array}$ \\
\hline & cg_Ftol & $\mathrm{Dble}$ & $1 e-6$ & Tolerance for the Conjugate Gradient minimization. \\
\hline & cg_stop & Int & 0 & $\begin{array}{l}\text { Stop condition for the Conjugate Gradient minimization of the cost } \\
\text { function } \chi(x) .0: C_{1}+C_{2}, 1: C_{1}, 2: C_{2} \text { with conditions } \\
C_{1}=\left|\chi^{n-1}-\chi^{n}\right|<\text { cg_Ftol }\left(1+\chi^{n}\right) \text {, } \\
C_{2}=\left\|x^{n-1}-x^{n}\right\|<\text { cg_Ftol }\left(1+\left\|x^{n}\right\|\right) \text {. See Sec. Sec. } 6.5\end{array}$ \\
\hline & cg_Weight & Int & 1 & $\begin{array}{l}\text { A weight factor used in the cost function } \chi \text { of the Conjugate } \\
\text { Gradient minimization. Increasing this parameter favors the fit of the } \\
\text { low frequency part of } \chi .1: \text { cg_weight }=1,2: \text { cg-weight }=1 / \text { Lfit, } 3 \text { : } \\
\text { cg-weight }=1 / \omega_{n} \text {. See Sec. Sec. } 6.5 \text {. }\end{array}$ \\
\hline & cg_pow & Int & 2 & $\begin{array}{l}\text { The exponent of the cost function } \chi \text { as }\left|X-X^{A n d}\right|^{\text {cg-pow }} \text { used in } \\
\text { Conjugate Gradient minimization. See Sec. } 6.5 \text {. }\end{array}$ \\
\hline \multirow[t]{2}{*}{$\circ$} & Hfile & Char & "hamiltonian" & File name to read/write bath parameters \\
\hline & SectorFile & Char & "sectors" & $\begin{array}{l}\text { File name to read/write sectors contributing to the state list. On } \\
\text { output it contains the QNs of the list of sectors contributing to the } \\
\text { evaluated spectrum. On input such list is used to reduce the sectors } \\
\text { analysis. See ed_sectors and ed_sectors_shift. }\end{array}$ \\
\hline$\circ$ & LOGfile & Int & 6 & $\begin{array}{l}\text { Fortran unit for the log output. Value must be in the range }[6: 999] \text {. } \\
6 \text { is the standard output. }\end{array}$ \\
\hline
\end{tabular}

\section{Appendix B: Main procedures}

In this appendix we report a list of all the procedures made accessible by either Fortan or Python API of the EDIpack library. As discussed in Sec. 7.2 all such procedures feature implicit MPI support, i.e. they are executed in parallel if the MPI framework has been initialized in the calling program.

In the first column we report the procedure names and the possible input arguments. In order to unify the naming conventions of the interfaces we use the prefix ?=ed_, edipack. to indicate, respectively, the Fortran and Python procedures. In the second column we briefly describe the purpose of the procedure and the nature of the arguments. The inputs in square brackets are optional.

The procedures are grouped with respect to the library module containing them, indicated in a single column at the beginning of each group.

Table 2: List of the EDIpack procedures.

\begin{tabular}{ll}
\hline \hline PROCEDURE (?=ed_, edipack.) & INFO \\
\hline \hline INPUT_VARS \\
\hline \hline ?read_input(File) & $\begin{array}{l}\text { Reads global input variables from the user indicated file File. } \\
\text { Argument: File is a character string of arbitrary length. If the } \\
\text { file does not exist in the calling directory a default one with } \\
\text { prefix "used." is produced using default values. The value of } \\
\text { each variable can be updated also from command line using the } \\
\text { syntax Variable=Value. }\end{array}$ \\
\hline \hline
\end{tabular}

\section{MAIN}


Table 2: List of the EDIpack procedures.

\begin{tabular}{ll}
\hline \hline PROCEDURE (?=ed, edipack.) & INFO \\
\hline \hline ?init_solver(Bath) & $\begin{array}{l}\text { Initializes the whole ED calculation, allocate global static } \\
\text { memory, perform compatibility checks and setup global variables. } \\
\text { Argument: Bath is a rank-1 dble array of dimension }\end{array}$ \\
& $\begin{array}{l}\text { Nb=?get_bath_dimension. For problems with Nsites inequivalent } \\
\text { atoms Bath is a rank-2 dble array of dimensions [Nsites,Nb]. On } \\
\text { Bath contains the bath parameters, either guessed from flat } \\
\text { density of states or read from the file Hfile.restart. }\end{array}$ \\
\hline ?solve(Bath, Hloc) & $\begin{array}{l}\text { Solve the quantum impurity problem using ED method. } \\
\text { Arguments: Bath is a rank-1 dble array of dimension } \\
\text { Nb=?get_bath_dimension. For problems with Nsites inequivalent } \\
\text { atoms Bath is a rank-2 dble array of dimensions [Nsites,Nb]. Hloc } \\
\text { is a rank-4 dble array of dimensions [Nspin, Nspin, Norb, Norb] or a } \\
\text { rank-5 of dimensions [Nsites, Nspin, Nspin, Norb, Norb]. Arguments } \\
\text { are not changed on output. }\end{array}$ \\
\hline \hline
\end{tabular}

\section{IO}

?get_sigma_matsubara(Func, [Nsites])

Returns the Matsubara self-energy function in Func. Argument: Func is a rank-5 cmplx array of dimensions

[Nspin, Nspin, Norb, Norb, Lmats]. If Nsites is present Func is a rank- 6 cmplx array of dimensions

[Nsites, Nspin, Nspin, Norb, Norb, Lmats] and the procedure returns the function for each inequivalent atom.

?get_sigma_realaxis(Func, [Nsites])

Returns the real-axis self-energy function in Func. Argument: Func is a rank- 5 cmplx array of dimensions [Nspin, Nspin, Norb, Norb, Lreal]. If Nsites is present Func is a rank-6 cmplx array of dimensions

[Nsites, Nspin, Nspin, Norb, Norb, Lreal] and the procedure returns the function for each inequivalent atom.

?get_gimp_matsubara(Func, [Nsites])

Returns the Matsubara impurity Green's function in Func. Argument: Func is a rank-5 cmplx array of dimensions [Nspin, Nspin, Norb, Norb, Lmats]. If Nsites is present Func is a rank- 6 cmplx array of dimensions

[Nsites, Nspin, Nspin, Norb, Norb, Lmats] and the procedure return. the function for each inequivalent atom.

?get_gimp_realaxis (Func, [Nsites])

Returns the real-axis impurity Green's function in Func. Argument: Func is a rank- 5 cmplx array of dimensions [Nspin, Nspin, Norb, Norb, Lreal]. If Nsites is present Func is a rank-6 cmplx array of dimensions

[Nsites, Nspin, Nspin, Norb, Norb, Lreal] and the procedure returns the function for each inequivalent atom.

?get_dens (Var, [Nsites])

Returns the impurity occupations in Var. Argument: Var is a rank-1 dble array of dimensions [Norb]. If Nsites is present var is a rank-2 dble array of dimensions [Nsites, Norb] and the procedure returns the value for each inequivalent atom.

?get_mag (Var, [Nsites])

Returns the impurity magnetization in Var. Argument: Var is a rank-1 dble array of dimensions [Norb]. If Nsites is present var is a rank-2 dble array of dimensions [Nsites, Norb] and the procedure returns the value for each inequivalent atom.

?get_docc(Var, [Nsites])

Returns the impurity double occupancy in Var. Argument: Var is a rank-1 dble array of dimensions [Norb]. If Nsites is present var is a rank-2 dble array of dimensions [Nsites, Norb] and the procedure returns the value for each inequivalent atom.

?get_eimp (Var, [Nsites])

Returns the impurity local energies components in Var. Argument: Var is a rank-1 dble array of dimensions [4]. If Nsites is present var is a rank-2 dble array of dimensions [Nsites, 4] and the procedure returns the value for each inequivalent atom. 
Table 2: List of the EDIpack procedures.

\begin{tabular}{ll}
\hline \hline PROCEDURE (?=ed_, edipack.) & INFO \\
\hline \hline ?get_doubles (Var, [Nsites]) & $\begin{array}{l}\text { Returns additional impurity double occupancies from } \\
\text { multi-orbital terms in Var. Argument: Var is a rank-1 dble array } \\
\text { of dimensions [4]. If Nsites is present var is a rank-2 dble array } \\
\text { of dimensions [Nsites, } 4 \text { ] and the procedure returns the value for } \\
\text { each inequivalent atom. }\end{array}$ \\
\hline \hline
\end{tabular}

\section{BATH}

?get_bath_dimension()

Returns the number $\mathrm{Nb}$ of bath parameters required to describe the quantum impurity problem, based on the value of different input variables, e.g. Norb, Nbath, bath_type. Output: Nb integer constant.

?set_Hreplica(Hloc)

?set_Hreplica(Hvec, LambdaVec)

Sets up the matrix basis and the initial guess for the variational bath parameters which determined the shape of the bath for bath_type=replica. The procedure accepts either the local non-interacting Hamiltonian Hloc or the Nsym matrix basis components Hvec and the corresponding variational parameters Lambdavec. See Sec. 6.4. Arguments: Hloc is a rank-4 dble array Lambdavec. See Sec. 6.4. Arguments: Hloc is a rank-4 dble array
of dimensions [Nspin, Nspin, Norb, Norb] or a rank-2 dble array of dimensions [Nspin*Norb,Nspin*Norb]. Hvec is a rank-5 dble array of dimensions [Nspin, Nspin,Norb, Norb, Nsym]. LambdaVec is a rank-1 dble array of dimensions Nsym or a rank-2 dble array of dimensions [Nsites, Nsym], for Nsites inequivalent atoms.

?get_bath_component_dimension(Type)

Returns the dimensions a rank-3 dble array should have to store a specific component of the bath. The output is a rank-1 int array $\operatorname{Ndim}(1: 3)$ of dimension 3. Argument: Type is a single character. Possible values are Type $=\mathrm{e}, \mathrm{v}, 1$, corresponding to energy, hybridization or lambda components.

?get_bath_component (Array, Bath, Type)

Returns in Array the specified components Type of the bath Bath. Arguments: Array a rank-3 dble array of dimensions $[\operatorname{Ndim}(1), \operatorname{Ndim}(2), \operatorname{Ndim}(3)]$ as returned by a call to ?get_bath_component_dimension(Type). Bath is a rank-1 dble array of dimension $\mathrm{Nb}=$ ?get_bath_dimension. Type=e, v, 1 is a single character.

?set_bath_component (Array, Bath, Type)

Sets the specified component Type in the bath Bath to Array. Arguments: Array a rank-3 dble array of dimensions $[\operatorname{Ndim}(1), \operatorname{Ndim}(2), \operatorname{Ndim}(3)]$ as returned by a call to ?get_bath_component_dimension(Type). Bath is a rank-1 dble array of dimension $\mathrm{Nb}=$ ?get_bath_dimension. Type=e, v, 1 is a single character.

?copy_bath_component (BathIN, BathOUT, Type)

Copies the specified component Type from the input bath BathIn to the output bath BathOut. Arguments: BathIn and BathOut are rank-1 dble array of dimension $\mathrm{Nb}=$ ?get_bath_dimension. Type=e, $\mathrm{v}, \mathrm{I}$ is a single character.

?spin_symmetrize_bath (Bath [, save])

Enforces spin symmetry in the bath Bath. Arguments: Bath is a rank-1 dble array of dimension $\mathrm{Nb}=$ ?get_bath_dimension. For problems with Nsites inequivalent atoms Bath is a rank-2 dble array of dimensions [Nsites,Nb]. save is a logical value used to optionally write out the output bath.

?orb_symmetrize_bath (Bath [, save])

Enforces symmetry among orbitals in the bath Bath. Arguments: Bath is a rank-1 dble array of dimension $\mathrm{Nb}=$ ?get_bath_dimension. For problems with Nsites inequivalent atoms Bath is a rank-2 dble array of dimensions [Nsites, $\mathrm{Nb}$ ]. save is a logical value used to optionally write out the output bath.

?orb_equality_bath (Bath, Indx [, save] )

Equals each orbital components in the bath Bath to be identical to those of the orbital Indx. Arguments: Bath is a rank-1 dble array of dimension $\mathrm{Nb}=$ ?get_bath_dimension. For problems with Nsites inequivalent atoms Bath is a rank-2 dble array of dimensions [Nsites,Nb]. Indx is a integer constant. save is a logical value used to optionally write out the output bath. 
Table 2: List of the EDIpack procedures.

\begin{tabular}{|c|c|}
\hline PROCEDURE (?=ed, , edipack.) & $I N F O$ \\
\hline ?ph_symmetrize_bath (Bath [, save] ) & $\begin{array}{l}\text { Enforces particle-hole symmetry in the bath Bath. Arguments: } \\
\text { Bath is a rank- } 1 \text { dble array of dimension } \mathrm{Nb}=\text { ?get_bath_dimension. } \\
\text { For problems with Nsites inequivalent atoms Bath is a rank- } 2 \\
\text { dble array of dimensions [Nsites, Nb]. save is a logical value used } \\
\text { to optionally write out the output bath. }\end{array}$ \\
\hline ?ph_trans_bath (Bath [, save] ) & $\begin{array}{l}\text { Perform a particle-hole transformation onto the bath Bath. } \\
\text { Arguments: Bath is a rank-1 dble array of dimension } \\
\mathrm{Nb}=\text { ?get_bath_dimension. For problems with Nsites inequivalent } \\
\text { atoms Bath is a rank-2 dble array of dimensions [Nsites,Nb]. save } \\
\text { is a logical value used to optionally write out the output bath. }\end{array}$ \\
\hline ?break_symmetry_bath (Bath,Field,Sign [, save]) & $\begin{array}{l}\text { Breaks spin symmetry in the bath Bath using a small field of } \\
\text { amplitude Field with sign Sign. The quantity Field*Sign is } \\
\text { added or subtracted, respectively for spin up and down, to the } \\
\text { local energies of each bath level. Arguments: Bath is a rank-1 } \\
\text { dble array of dimension Nb=?get_bath_dimension. For problems } \\
\text { with Nsites inequivalent atoms Bath is a rank-2 dble array of } \\
\text { dimensions [Nsites, Nb]. Field, Sign are dble constant. save is a } \\
\text { logical value used to optionally write out the output bath. }\end{array}$ \\
\hline
\end{tabular}

\section{BATH_FIT}

?chi2_fitgf (Func, Bath, Hloc, ispin [, iorb])

Optimizes the bath Bath according to the user-provided function Func, using a Conjugate Gradient minimization of the cost function $\chi$, see Sec. 6.5. Argument: Func is a rank-5 cmplx array of dimensions [Nspin, Nspin, Norb, Norb, :]. For problems with Nsites inequivalent atoms Func is a rank- $6 \mathrm{cmplx}$ array of dimensions [Nsites, Nspin, Nspin, Norb, Norb, : ]. Bath is a rank-1 dble array of dimension $\mathrm{Nb}=$ ?get_bath_dimension. or a rank-2 dble array of dimensions [Nsites, Nb]. Hloc is a rank-4 dble array of dimensions [Nspin, Nspin, Norb, Norb] or a rank-5 of dimensions [Nsites, Nspin, Nspin, Norb, Norb]. ispin is an int constant indicating the spin components to fit. The optional argument iorb indicate the orbital components to fit. If not passed all orbitals are fitted.

\begin{tabular}{ll}
\hline \hline ?set_suffix(ilat,pads) & AUX_FUNX \\
\hline ?reset_suffix() & $\begin{array}{l}\text { Sets an additional suffix for the output files of the EDIpack } \\
\text { library. Arguments: ilat is an integer constant. pads is an } \\
\text { integer constant indicating the number of zero padding of the } \\
\text { integer ilat in the suffix. }\end{array}$ \\
\hline ?search_variable(var,ntmp, bool) & $\begin{array}{l}\text { Resets the suffix for the output files of EDIpack to the default } \\
\text { value }\end{array}$ \\
\hline & $\begin{array}{l}\text { Varies the variable var so that the conjugated variable ntmp is } \\
\text { equal to the input value nread up to an error nerr. Arguments: } \\
\text { var, ntmp are dble constants. bool is a logical constant indicating } \\
\text { the success of the variation. }\end{array}$ \\
\hline
\end{tabular}

\section{References}

[1] H. Lin, J. Gubernatis, Exact diagonalization methods for quantum systems, Computers in Physics 7 (4) (1993) $400-407$. URL https ://doi.org/10.1063/1.4823192

[2] A. C. Hewson, The Kondo Problem to Heavy Fermions, Cambridge University Press, New York, N.Y., 1993.

[3] G. Kotliar, D. Vollhardt, Strongly correlated materials: Insights from dynamical mean-field theory, Physics Today 57 (3) (2004) 53-59.

URL https://doi.org/10.1063/1.1712502

[4] A. Georges, G. Kotliar, W. Krauth, M. J. Rozenberg, Dynamical mean-field theory of strongly correlated fermion systems and the limit of infinite dimensions, Rev. Mod. Phys. 68 (1996) 13-125. doi:10.1103/RevModPhys.68.13.

URL https ://link. aps.org/doi/10.1103/RevModPhys.68.13 
[5] A. Georges, Strongly correlated electron materials: Dynamical mean-field theory and electronic structure, AIP Conference Proceedings 715 (1) (2004) 3-74. doi:10.1063/1.1800733.

URL https://aip.scitation.org/doi/abs/10.1063/1.1800733

[6] F. Lechermann, A. Georges, A. Poteryaev, S. Biermann, M. Posternak, A. Yamasaki, O. K. Andersen, Dynamical meanfield theory using Wannier functions: A flexible route to electronic structure calculations of strongly correlated materials, Phys. Rev. B 74 (12) (2006) 125120. doi:10.1103/PhysRevB.74.125120.

URL http://link.aps.org/abstract/PRB/v74/e125120

[7] M. Potthoff, Self-energy-functional approach to systems of correlated electrons, The European Physical Journal B Condensed Matter and Complex Systems 32 (4) (2003) 429-436. doi:10.1140/epjb/e2003-00121-8. URL https://doi.org/10.1140/epjb/e2003-00121-8

[8] D. Sénéchal, The variational cluster approximation for hubbard models: Practical implementation, in: 2008 22nd International Symposium on High Performance Computing Systems and Applications, 2008, pp. 9-15.

[9] M. Potthoff, Static and dynamic variational principles for strongly correlated electron systems, AIP Conference Proceedings 1419 (1) (2011) 199-258. doi:10.1063/1.3667325. URL https://aip.scitation.org/doi/abs/10.1063/1.3667325

[10] M. Nuss, E. Arrigoni, M. Aichhorn, W. von der Linden, Variational cluster approach to the single impurity anderson model (2011). arXiv:1110.4533.

[11] B. Bauer, L. D. Carr, H. G. Evertz, A. Feiguin, J. Freire, S. Fuchs, L. Gamper, J. Gukelberger, E. Gull, S. Guertler, A. Hehn, R. Igarashi, S. V. Isakov, D. Koop, P. N. Ma, P. Mates, H. Matsuo, O. Parcollet, G. Pawlowski, J. D. Picon, L. Pollet, E. Santos, V. W. Scarola, U. Schollwöck, C. Silva, B. Surer, S. Todo, S. Trebst, M. Troyer, M. L. Wall, P. Werner, S. Wessel, The ALPS project release 2.0: open source software for strongly correlated systems, Journal of Statistical Mechanics: Theory and Experiment 2011 (05) (2011) P05001. doi:10.1088/1742-5468/2011/05/p05001. URL https://doi.org/10.1088/1742-5468/2011/05/p05001

[12] O. Parcollet, M. Ferrero, T. Ayral, H. Hafermann, I. Krivenko, L. Messio, P. Seth, Triqs: A toolbox for research on interacting quantum systems, Computer Physics Communications 196 (2015) 398-415. doi:https://doi.org/10.1016/ j.cpc.2015.04.023.

URL https://www.sciencedirect.com/science/article/pii/S0010465515001666

[13] E. Gull, A. J. Millis, A. I. Lichtenstein, A. N. Rubtsov, M. Troyer, P. Werner, Continuous-time monte carlo methods for quantum impurity models, Rev. Mod. Phys. 83 (2011) 349-404. doi:10.1103/RevModPhys.83.349.

URL https://link.aps.org/doi/10.1103/RevModPhys.83.349

[14] A. N. Rubtsov, V. V. Savkin, A. I. Lichtenstein, Continuous-time quantum monte carlo method for fermions, Phys. Rev. B 72 (2005) 035122. doi:10.1103/PhysRevB.72.035122. URL https://link.aps.org/doi/10.1103/PhysRevB.72.035122

[15] K. Haule, Quantum monte carlo impurity solver for cluster dynamical mean-field theory and electronic structure calculations with adjustable cluster base, Phys. Rev. B 75 (15) (2007) 155113. doi:10.1103/PhysRevB.75.155113. URL http://link.aps.org/abstract/PRB/v75/e155113

[16] P. Seth, I. Krivenko, M. Ferrero, O. Parcollet, Triqs/cthyb: A continuous-time quantum monte carlo hybridisation expansion solver for quantum impurity problems, Computer Physics Communications 200 (2016) 274-284. doi:https: //doi.org/10.1016/j.cpc.2015.10.023. URL https://www.sciencedirect.com/science/article/pii/S001046551500404X

[17] M. Wallerberger, A. Hausoel, P. Gunacker, A. Kowalski, N. Parragh, F. Goth, K. Held, G. Sangiovanni, w2dynamics: Local one- and two-particle quantities from dynamical mean field theory, Computer Physics Communications 235 (2019) 388-399. doi:https://doi.org/10.1016/j.cpc.2018.09.007.

URL https://www.sciencedirect.com/science/article/pii/S0010465518303217

[18] R. Žitko, T. Pruschke, Energy resolution and discretization artifacts in the numerical renormalization group, Phys. Rev. B 79 (2009) 085106. doi:10.1103/PhysRevB.79.085106. URL https://link.aps.org/doi/10.1103/PhysRevB.79.085106

[19] R. Bulla, T. A. Costi, D. Vollhardt, Finite-temperature numerical renormalization group study of the Mott transition, Phys. Rev. B 64 (4) (2001) 045103. doi:10.1103/PhysRevB.64.045103.

[20] R. Bulla, T. A. Costi, T. Pruschke, Numerical renormalization group method for quantum impurity systems, Rev. Mod. Phys. 80 (2) (2008) 395. doi:10.1103/RevModPhys.80.395. URL http://link.aps.org/abstract/RMP/v80/p395

[21] S. R. White, Density matrix formulation for quantum renormalization groups, Phys. Rev. Lett. 69 (19) (1992) $2863-2866$. doi:10.1103/PhysRevLett.69.2863.

[22] K. A. Hallberg, Density-matrix algorithm for the calculation of dynamical properties of low-dimensional systems, Phys. Rev. B 52 (14) (1995) R9827-R9830. doi:10.1103/PhysRevB.52.R9827.

[23] D. J. García, K. Hallberg, M. J. Rozenberg, Dynamical mean field theory with the density matrix renormalization group, Phys. Rev. Lett. 93 (2004) 246403. doi:10.1103/PhysRevLett.93.246403. URL https://link.aps.org/doi/10.1103/PhysRevLett.93.246403

[24] U. Schollwöck, The density-matrix renormalization group, Rev. Mod. Phys. 77 (1) (2005) 259. doi:10.1103/RevModPhys. 77.259 .

URL http://link.aps.org/abstract/RMP/v77/p259

[25] F. A. Wolf, A. Go, I. P. McCulloch, A. J. Millis, U. Schollwöck, Imaginary-time matrix product state impurity solver for dynamical mean-field theory, Phys. Rev. X 5 (2015) 041032. doi:10.1103/PhysRevX.5.041032. URL https://link.aps.org/doi/10.1103/PhysRevX.5.041032 
[26] M. Caffarel, W. Krauth, Exact diagonalization approach to correlated fermions in infinite dimensions: Mott transition and superconductivity, Phys. Rev. Lett. 72 (1994) 1545-1548. doi:10.1103/PhysRevLett.72.1545.

URL https://link.aps.org/doi/10.1103/PhysRevLett.72.1545

[27] A. Dolfen, Massively parallel exact diagonalization of strongly correlated systems, Master's thesis, Forschungszentrum Juelich (2006). URL https://www.fz-juelich.de/cae/servlet/contentblob/1040316/publicationFile/23068/Dolfen.pdf

[28] C. A. Perroni, H. Ishida, A. Liebsch, Exact diagonalization dynamical mean-field theory for multiband materials: Effect of coulomb correlations on the fermi surface of na ${ }_{0.3} \mathrm{coO}_{2}$, Phys. Rev. B 75 (2007) 045125. doi:10.1103/PhysRevB.75.045125. URL https://link.aps.org/doi/10.1103/PhysRevB.75.045125

[29] M. Capone, L. de' Medici, A. Georges, Solving the dynamical mean-field theory at very low temperatures using the lanczos exact diagonalization, Phys. Rev. B 76 (2007) 245116. doi:10.1103/PhysRevB.76.245116. URL https://link.aps.org/doi/10.1103/PhysRevB.76.245116

[30] C. Weber, A. Amaricci, M. Capone, P. B. Littlewood, Augmented hybrid exact-diagonalization solver for dynamical mean field theory, Phys. Rev. B 86 (2012) 115136. doi:10.1103/PhysRevB.86.115136. URL http://link.aps.org/doi/10.1103/PhysRevB.86.115136

[31] Y. Lu, M. W. Haverkort, Exact diagonalization as an impurity solver in dynamical mean field theory, The European Physical Journal Special Topics 226 (11) (2017) 2549-2564. doi:10.1140/epjst/e2017-70042-4. URL https://doi.org/10.1140/epjst/e2017-70042-4

[32] H. Fehske, R. Schneider, A. Weisse (Eds.), Exact Diagonalization Techniques, Springer Berlin Heidelberg, Berlin, Heidelberg, 2008, pp. 529-544.

URL https://doi.org/10.1007/978-3-540-74686-7_18

[33] A. W. Sandvik, Computational studies of quantum spin systems, AIP Conference Proceedings 1297 (1) (2010) $135-338$. arXiv:https://aip.scitation.org/doi/pdf/10.1063/1.3518900, doi:10.1063/1.3518900. URL https://aip.scitation.org/doi/abs/10.1063/1.3518900

[34] T. Siro, A. Harju, Exact diagonalization of the hubbard model on graphics processing units, Computer Physics Communications 183 (9) (2012) 1884-1889. doi:https://doi.org/10.1016/j.cpc.2012.04.006. URL https://www.sciencedirect.com/science/article/pii/S0010465512001452

[35] U. Borštnik, J. VandeVondele, V. Weber, J. Hutter, Sparse matrix multiplication: The distributed block-compressed sparse row library, Parallel Computing 40 (5) (2014) 47-58. doi:https://doi.org/10.1016/j.parco.2014.03.012. URL https://www.sciencedirect.com/science/article/pii/S0167819114000428

[36] T. Siro, A. Harju, Exact diagonalization of quantum lattice models on coprocessors, Computer Physics Communications 207 (2016) 274-281. doi:https://doi.org/10.1016/j.cpc.2016.07.018. URL https://www.sciencedirect.com/science/article/pii/S0010465516302065

[37] M. Sharma, M. Ahsan, Organization of the hilbert space for exact diagonalization of hubbard model, Computer Physics Communications 193 (2015) 19-29. doi:https://doi.org/10.1016/j.cpc.2015.03.014. URL https://www.sciencedirect.com/science/article/pii/S0010465515001137

[38] G. Kotliar, S. Y. Savrasov, K. Haule, et al., Electronic structure calculations with dynamical mean-field theory, Rev. Mod. Phys. 78 (3) (2006) 865. doi:10.1103/RevModPhys.78.865.

[39] C. Lanczos, An iteration method for the solution of the eigenvalue problem of linear differential and integral operators, J. Res. Natl. Bur. Stand. B 45 (1950) 255-282. doi:10.6028/jres.045.026.

[40] W. E. ARNOLDI, The principle of minimized iterations in the solution of the matrix eigenvalue problem, Quarterly of Applied Mathematics 9 (1) (1951) 17-29. URL http://www.jstor.org/stable/43633863

[41] A.Krylov, De la résolution numérique de l'équation servant à déterminer dans des questions de mécanique appliquée les fréquences de petites oscillations des systèmes matériels, Bulletin de l'Académie des Sciences de l'URSS 5 (5) (1931) 491-539. URL http://mi.mathnet.ru/izv5215

[42] E. Polizzi, Density-matrix-based algorithm for solving eigenvalue problems, Phys. Rev. B 79 (2009) 115112. doi:10.1103/ PhysRevB.79.115112. URL https://link.aps.org/doi/10.1103/PhysRevB.79.115112

[43] A. Georges, L. de' Medici, J. Mravlje, Strong Correlations from Hund's Coupling., Annu. Rev. Condens. Matter Phys. 45 (2013) 137-178.

[44] R. Lehoucq, D. Sorensen, C. Yang, ARPACK Users' Guide: Solution of Large-scale Eigenvalue Problems with Implicitly Restarted Arnoldi Methods, Software, Environments, Tools, Society for Industrial and Applied Mathematics, 1998. URL https://books.google.it/books?id=iMUea23N_CQC

[45] K. J. "Maschhoff, D. C. Sorensen, P_arpack: An efficient portable large scale eigenvalue package for distributed memory parallel architectures, in: J. "Waśniewski, J. Dongarra, K. Madsen, D. Olesen (Eds.), Applied Parallel Computing Industrial Computation and Optimization, Springer Berlin Heidelberg, Berlin, Heidelberg, 1996, pp. 478-486.

[46] E. Koch, G. Sangiovanni, O. Gunnarsson, Sum rules and bath parametrization for quantum cluster theories, Phys. Rev. B 78 (2008) 115102. doi:10.1103/PhysRevB.78.115102. URL https://link.aps.org/doi/10.1103/PhysRevB.78.115102

[47] C. Taranto, G. Sangiovanni, K. Held, M. Capone, A. Georges, A. Toschi, Signature of antiferromagnetic long-range order in the optical spectrum of strongly correlated electron systems, Phys. Rev. B 85 (2012) 085124. doi:10.1103/PhysRevB. 85.085124 . URL https://link.aps.org/doi/10.1103/PhysRevB.85.085124 
[48] W. H. Press, B. P. Flannery, S. A. Teukolsky, W. T. Vetterling, Numerical Recipes in FORTRAN 77: The Art of Scientific Computing, 2nd Edition, Cambridge University Press, 1992.

URL http://www . worldcat.org/isbn/052143064X

[49] Message Passing Interface Forum, MPI: A Message-Passing Interface Standard, Version 2.2, High Performance Computing Center Stuttgart (HLRS), 2009.

URL http://www.mpi-forum.org/docs/mpi-2.2/mpi22-report.pdf 